\title{
Characterization and Analysis of Volatile Fingerprint
}

\section{of 13 Different Commercial Essential Oils with GC-}

\section{MS and Chemical Gas Sensors}

\author{
Estefanía Núñez-Carmona ${ }^{1}$, Marco Abbatangelo ${ }^{1, *}$ and Veronica Sberveglieri ${ }^{2,3}$ \\ 1 University of Brescia, Dep. Information Engineering, Brescia, Via Branze, 38, 25123 Brescia (BS), Italy \\ e.nunezcarmona@unibs.it \\ 2 CNR-IBBR, Institute of Bioscience and Bioresources; via Madonna del Piano, 10, 50019 Sesto Fiorentino \\ (FI), Italy, veronica.sberveglieri@ibbr.cnr.it \\ 3 NANO SENSOR SYSTEMS, NASYS spin-off University of Brescia, Dep. Information Engineering, Brescia, \\ Via Camillo Brozzoni, 9, 25125 Brescia (BS), Italy. \\ * Correspondence: m.abbatangelo@unibs.it;
}

\begin{abstract}
Essential oils are mixtures of compounds obtained from plants, including flowers, roots, bark, leaves, seeds,peel, fruits, wood, that have risen up in the last decades thanks to their beneficial properties as antibacterial, antifungal and anti-inflammatory agents. The aim of this study was to characterize and analyze 13 different commercial essential oils with two different techniques. The first is GC-MS, coupled with SPME, thanks to which 204 different VOCs have been identified. The results show that a total of 95 compounds was found only in one oil, while the others were found with different frequencies in many of them. The most represented class is that of terpenes, as widely reported in literature. The second technique is based on an array of chemical gas sensors. This system was used to investigate whether sensors are able to identify these products. It turned out that basil, cinnamon and carnation are the most identifiable oils with different number and typology of sensors, especially tin oxide and copper oxide nanowires, while cayeput and thyme are more mistakable samples. Thanks to this detailed study, it has been possible to reach and obtain novel insights for the future development of this type of research.
\end{abstract}

Keywords: essential oils; VOCs; GC-MS SPME; electronic nose; nanowire gas sensors; ANOVA.

\section{Introduction}

Essential oils (EOs) are complex mixtures of compounds extracted as secondary metabolites from aromatic plants. Since they are hydrophobic and have often a density lower than that of water, are lipophilic and soluble in organic solvents [1]. EOs can be obtained in different ways, that can be grouped together in two main classes: classical methods, such as hydro distillation [2] and cold pressing [3], and innovative techniques, as supercritical fluid extraction [4,5]. The extraction is regulated by International Standards Organization (ISO) [6].

EOs were well known since ancient civilizations, such as the Egyptians and the Persians, for their antiseptic, fungicidal and antimicrobial properties, that have been preserved until today [7]. All these characteristics are back to be investigated in recent years as they give the EOs the potential to be used in many fields. Food preservation is one and green materials are gaining attention for the development of biodegradable packaging materials. Hence, the incorporation of EOs in new films brings two advantages, i.e. antimicrobial activity and physicochemical properties improvement [813]. Related to this field, EOs are promising substances as antibiotic alternatives in animal production 
like poultry and swine, although the metabolisms and the mechanisms of their activities should be better understood [14-16]. In the last years, several studies have been carried out in order to understand the antimicrobial effects of different oils on microorganisms and foodborne pathogens [17]. As examples, the effects of cardamom, cumin, and dill weed EOs have been investigated against Campylobacter spp. [18]; basil, oregano and thyme were effective against E. coli, Salmonella enteritidis, L. monocytogenes [19,20]; oils extracted from dill herb (leaves and seed) were used for their antifungal and antimicrobial capability towards Aspergillus genus and Staphylococcus aureus, Escherichia coli, Pseudomonas aeruginosa, respectively [21]; coriander has shown the higher antimicrobial activity among ten Apiaceous fruit against E. coli and Bordetella bronchiseptica [22]; six different juniper species were examined to evaluate which were the effects on different bacteria [23].

Finally, among all the benefits of EOs, it is important to remember also their medicinal and therapeutic potentials. They are antioxidant and anti-aging [24], cancer preventive and antiinflammatory [25].

On the other hand, in the last years a high interest has risen up regarding the use of chemical sensors to perform rapid and cost-efficient analysis in many different fields of application. The main fields of application for this kind of technology are environmental [26-29] and health monitoring [3031], followed up by quality control in foods [32-33]. Nowadays a lot of different types of chemical sensor are on the market, one of the most used categories are Metal Oxide (MOX) sensors that bases their working principle in the semiconductor characteristics of these materials. Nanowire MOX gas sensor (NWs) [34] are one of the many different semiconductor chemical sensors but in particular this kind of technology merge also the main characteristics of the nanostructured materials, as high length to width ratio, which reduce the threshold of detection of some compounds, and long-term stability for sustained operation. The promising performances NWs have been extensively reported in literature in many different fields as environmental and health monitoring [35-37] and food quality control [38-40].

The aim of this work was to characterize and analyze the set of volatile organic compounds (VOCs) of thirteen different commercial EOs. The study proceeded in two steps. First of all, a Gas Cromatograph with Mass Spectometer (GC-MS) has been used in order to identify the compounds of the samples and to understand which were common and which distinctive of the specific sample. This technique is widely used for this purpose [41,42]. GC-MS has been coupled with Solid Phase Micro Extraction (SPME) technique. Secondly, an analysis with array of nanowire gas sensors placed in an innovative device called Small Sensor System (S3) was carried out.

\section{Results \& Discussion}

\subsection{GC-MS Analysis}

GC-MS analysis, of the 13 EOs samples, led to the identification of 204 different VOCs. In Table 1, they are reported for increasing retention time (Rt), starting from $2.04 \mathrm{~min}$ to $58.61 \mathrm{~min}$. The samples to which the compounds belong and the abundance are indicated. Abundance has been calculated as the mean value of the two replicas of the peak areas obtained from the chromatogram, every samples was analyzed in two replicas. Values are scaled by a factor of $10^{\wedge}-6$. The name of most compounds has been found mainly in two References [43-44].

Table 1. List of VOCs found in the 13 EOs with retention time (Rt) and pick areas. Abundance of the area is the mean of two replicas. Values are scaled by a factor of $10^{\wedge}-6$. B=basil, $\mathrm{C}=$ cinnamon, Card=cardamom, $\mathrm{Cay}=$ cayeput, $\mathrm{Co}=$ coriander, Carn=carnation, J=Jupiter, Ni=niaouly, Nu=nutmeg, $\mathrm{O}=\mathrm{oregan}, \mathrm{R}=$ rosemary, $\mathrm{M}=$ mustard, $\mathrm{T}=$ thyme. 
3 of 19

\begin{tabular}{|c|c|c|c|c|c|c|c|c|c|c|c|c|c|c|}
\hline & & $B$ & $\mathrm{Ci}$ & Card & Cay & Co & Carn & $J$ & $N i$ & $N u$ & O & $R$ & $M$ & $T$ \\
\hline 2.04 & N,N-Dimethyl-3-Buten-1-amine & 0 & 0 & 0 & 0 & 0,266 & 0 & 0 & 0 & 0 & 0 & 0 & 0 & 0 \\
\hline 2.08 & Methoxyethylene & 0 & 0 & 4,002 & 0 & 0 & 0 & 0 & 0 & 0 & 0 & 0 & 0 & 0 \\
\hline 2.70 & Cyclopropylamine & 0 & 0 & 0 & 0 & 0 & 0 & 0 & 0 & 0 & 0 & 0 & 68,866 & 0 \\
\hline 4.51 & 1R- $\alpha$-Pinene & 0 & 246,652 & 209,99 & 120,922 & 688,009 & 0 & $\begin{array}{r}2395,5 \\
15\end{array}$ & 127,54 & 973,149 & 229,777 & 1353,925 & 0 & $\begin{array}{r}492,95 \\
5\end{array}$ \\
\hline 5.14 & (2-Methyloctyl)benzene & 0 & 0 & 0 & 0 & 0 & 3,955 & 0 & 0 & 0 & 0 & 0 & 0 & 0 \\
\hline 5.15 & $\begin{array}{l}\text { 2-Methyl-2-(2,2,4,4- } \\
\text { tetramethylpentyl)-aziridine }\end{array}$ & 0 & 0 & 0 & 0 & 0 & 0 & 0 & 0 & 0 & 0 & 0 & 0,477 & 0 \\
\hline 5.57 & Camphene & 0 & 79,731 & 0 & 28,776 & 153,889 & 0 & 0 & 28,37 & 0 & 59,521 & 697,084 & 0 & $\begin{array}{r}183,77 \\
4\end{array}$ \\
\hline 7.21 & $\beta$-Pinene & 0 & 71,616 & 31,439 & 154,377 & 48,345 & 0 & $\begin{array}{r}128,50 \\
3\end{array}$ & 161,683 & 573,457 & 336,578 & 1088,569 & 0 & 52,663 \\
\hline 8.14 & $\beta$-Phellandrene & 0 & 28,348 & 625,935 & 0 & 0 & 0 & $\begin{array}{r}163,95 \\
4\end{array}$ & 0 & 135,488 & 0 & 0 & 0 & 0 \\
\hline 8.37 & $\begin{array}{l}\text { 3-Isopropenyl-5,5-dimethyl- } \\
\text { cyclopentene }\end{array}$ & 0 & 0 & 0 & 0 & 0 & 0 & 0 & 0 & $\begin{array}{r}1688,54 \\
8\end{array}$ & 0 & 0 & 0 & 0 \\
\hline 8.54 & 1-Methoxy-2-propanol & 0 & 8,844 & 0 & 19,662 & 8,435 & 8,021 & 0 & 17,313 & 0 & 13,46 & 0 & 5,133 & 0 \\
\hline 8.66 & $\begin{array}{c}1-(1- \\
\text { Methoxymethoxyethyl)cyclohexe } \\
\text { ne }\end{array}$ & 0 & 0 & 0 & 0 & 0 & 0 & 0 & 0 & 0 & 0 & 0 & 0 & 23,654 \\
\hline 9.54 & Diallyl sulfide & 0 & 0 & 0 & 0 & 0 & 0 & 0 & 0 & 0 & 0 & 0 & 156,129 & 0 \\
\hline 9.91 & 3-Heptanone & 5,741 & 0 & 6,038 & 10,318 & 9,88 & 19,626 & 0 & 10,655 & 0 & 0 & 0 & 0 & 7,372 \\
\hline $\begin{array}{c}10.2 \\
3\end{array}$ & $\alpha$-Phellandrene & 0 & 253,238 & 0 & 0 & 0 & 0 & 0 & 0 & 71,01 & 0 & 0 & 0 & 0 \\
\hline $\begin{array}{c}10.6 \\
3\end{array}$ & $\beta$-Myrcene & 0 & 0 & 84,547 & 62,101 & 26,488 & 0 & $\begin{array}{r}714,58 \\
1\end{array}$ & 65,17 & 0 & 477,578 & 0 & 0 & $\begin{array}{r}230,67 \\
1\end{array}$ \\
\hline $\begin{array}{c}11.4 \\
2\end{array}$ & $\alpha$-Ethylcaproaldehyde & 1,574 & 0 & 0 & 0 & 0 & 1,227 & 0 & 0 & 0 & 0 & 0 & 3,096 & 0 \\
\hline $\begin{array}{c}11.8 \\
8\end{array}$ & D-Limonene & 0 & 82,25 & 0 & 0 & 412,02 & 0 & $\begin{array}{r}2041,2 \\
69\end{array}$ & 0 & 0 & 0 & 0 & 0 & $\begin{array}{r}301,65 \\
5\end{array}$ \\
\hline $\begin{array}{c}11.9 \\
7\end{array}$ & 1,4-Dimethyl-4-vinylcyclohexene & 0 & 0 & 0 & 0 & 0 & 0 & 0 & 0 & 419,344 & 0 & 0 & 0 & 0 \\
\hline $\begin{array}{c}12.0 \\
5\end{array}$ & $\beta$-Terpineol & 0 & 0 & 0 & 0 & 0 & 0 & 0 & 0 & 0 & 156,956 & 0 & 0 & 0 \\
\hline $\begin{array}{c}12.1 \\
2\end{array}$ & Eucalyptol & 296,274 & 184,082 & 2006,264 & $\begin{array}{r}2884,90 \\
4\end{array}$ & 0 & 0 & 0 & 2977,09 & 309,084 & 0 & 2826,559 & 0 & 0 \\
\hline $\begin{array}{c}12.3 \\
6\end{array}$ & Methoxybutanol & 0 & 0 & 0 & 0 & 0 & 8,397 & 0 & 0 & 0 & 0 & 0 & 7,902 & 0 \\
\hline $\begin{array}{c}12.9 \\
0\end{array}$ & Thiazole & 0 & 0 & 0 & 0 & 0 & 0 & 0 & 0 & 0 & 0 & 0 & 30,169 & 0 \\
\hline $\begin{array}{c}13.3 \\
0\end{array}$ & 1-[1-Cycloazapropyl]-n-Octane & 8,728 & 6,491 & 0 & 0 & 8,113 & 6,295 & 7,071 & 0 & 14,781 & 8,788 & 0 & 0 & 0 \\
\hline $\begin{array}{c}13.4 \\
3\end{array}$ & 8-Methylenepentadecane & 0 & 0 & 0 & 39,637 & 0 & 0 & 0 & 46,818 & 0 & 0 & 36,451 & 0 & 0 \\
\hline $\begin{array}{c}13.7 \\
1\end{array}$ & 1-Decylaziridine & 0 & 0 & 0 & 0 & 0 & 0 & 0 & 0 & 0 & 0 & 0 & 0 & 6,153 \\
\hline $\begin{array}{c}14.1 \\
3\end{array}$ & $\gamma$-Terpinene & 0 & 48,044 & 0 & 25,79 & 82,952 & 0 & 63,32 & 27,452 & 463,12 & 559,739 & 137,353 & 0 & 0 \\
\hline $\begin{array}{c}14.3 \\
9\end{array}$ & 1-Undecene & 0 & 0 & 0 & 0 & 0 & 1,007 & 0 & 0 & 0 & 0 & 0 & 0 & 0 \\
\hline $\begin{array}{c}14.7 \\
3\end{array}$ & $\begin{array}{l}\mathrm{N}-[(4-H y d r o x y) h y d r o c i n n a m o y l]- \\
\text { Benzeneethanamine }\end{array}$ & 0 & 24,029 & 0 & 0 & 0 & 0 & 0 & 0 & 0 & 0 & 0 & 0 & 0 \\
\hline $\begin{array}{c}14.8 \\
2\end{array}$ & $\beta$-Ocimene & 0 & 0 & 0 & 44,073 & 0 & 0 & 0 & 47,878 & 0 & 0 & 29,047 & 0 & 0 \\
\hline $\begin{array}{c}15.0 \\
8\end{array}$ & 1-Butoxy-2-ethylhexane & 0 & 0 & 0 & 0 & 0 & 0 & 0 & 0 & 0 & 0 & 0 & 2,564 & 0 \\
\hline $\begin{array}{c}15.1 \\
9\end{array}$ & p-Cymene & 8,272 & 0 & 0 & 0 & 0 & 12,989 & 0 & 0 & 0 & 0 & 0 & 0 & 0 \\
\hline $\begin{array}{c}15.2 \\
7\end{array}$ & M-Cymene & 0 & 339,522 & 93,37 & 63,366 & 0 & 0 & 91,267 & 66,913 & 216,747 & 0 & 333,558 & 0 & 0 \\
\hline $\begin{array}{c}15.3 \\
8\end{array}$ & o-Cymene & 0 & 0 & 0 & 0 & 584,098 & 0 & 0 & 0 & 0 & $\begin{array}{r}1201,20 \\
3\end{array}$ & 0 & 0 & $\begin{array}{r}2115,9 \\
68\end{array}$ \\
\hline $\begin{array}{c}15.7 \\
1\end{array}$ & (+)-4-Carene & 0 & 7,473 & 0 & 18,686 & 5,163 & 0 & $\begin{array}{r}235,03 \\
4\end{array}$ & 0 & 868,631 & 180,754 & 194,837 & 0 & 0 \\
\hline
\end{tabular}


4 of 19

\begin{tabular}{|c|c|c|c|c|c|c|c|c|c|c|c|c|c|c|}
\hline $\begin{array}{c}15.8 \\
5\end{array}$ & 1-Azepan-1-yl-2-bromethanone & 0 & 0 & 0 & 0 & 0 & 0 & 0 & 0 & 0 & 0 & 0 & 9,566 & 0 \\
\hline $\begin{array}{c}15.8 \\
8\end{array}$ & $\begin{array}{l}\text { Lactic acid, monoanhydride with } \\
\text { 1-butaneboronic acid, cyclic ester }\end{array}$ & 76,743 & 37,209 & 120,834 & 103,031 & 85,449 & 84,357 & 0 & 130,325 & 0 & 0 & 0 & 61,796 & 0 \\
\hline $\begin{array}{c}16.8 \\
7\end{array}$ & Tridecane & 0 & 21,214 & 0 & 0 & 0 & 32,486 & 0 & 0 & 2,202 & 0 & 0 & 0 & 0 \\
\hline $\begin{array}{c}17.5 \\
9\end{array}$ & 3-Butyl-cyclopenatnone & 0 & 0 & 0 & 0 & 0 & 0 & 0 & 0 & 0 & 0 & 0 & 12,456 & 0 \\
\hline $\begin{array}{c}17.8 \\
3\end{array}$ & 2-Heptanol & 0 & 0 & 0 & 0 & 0 & 5,36 & 0 & 0 & 0 & 0 & 0 & 0 & 0 \\
\hline $\begin{array}{c}17.9 \\
9\end{array}$ & 1-Adamantanol & 0 & 0 & 0 & 0 & 0 & 0 & 0 & 0 & 2,739 & 0 & 0 & 0 & 0 \\
\hline $\begin{array}{c}18.3 \\
1\end{array}$ & Methyl Heptenone & 0 & 0 & 44,593 & 0 & 0 & 0 & 0 & 0 & 0 & 0 & 0 & 0 & 0 \\
\hline $\begin{array}{c}18.4 \\
1\end{array}$ & Nonanenitrile & 22,089 & 17,107 & 0 & 25,576 & 25,716 & 30,884 & 18,898 & 26,007 & 14,007 & 18,511 & 29,649 & 18,683 & 14,803 \\
\hline $\begin{array}{c}19.0 \\
8\end{array}$ & Hexyl formate & 74,396 & 107,316 & 127,484 & 104,903 & 95,14 & 119,112 & 93,84 & 109,322 & 96,787 & 103,847 & 117,823 & 109,848 & $\begin{array}{r}103,02 \\
5\end{array}$ \\
\hline $\begin{array}{c}19.6 \\
2\end{array}$ & Neo-allo-ocimene & 0 & 5,482 & 0 & 0 & 0 & 0 & 6,401 & 0 & 0 & 0 & 3,197 & 0 & 0 \\
\hline $\begin{array}{c}20.0 \\
4\end{array}$ & Ethyl glycidyl ether & 0 & 5,741 & 2,276 & 0 & 2,349 & 4,418 & 0 & 1,793 & 0 & 6,816 & 0 & 1,71 & 0 \\
\hline $\begin{array}{c}20.1 \\
7\end{array}$ & cis-p-Mentha-2,8-dien-1-ol & 0 & 0 & 0 & 0 & 0 & 0 & 0 & 0 & 4,856 & 0 & 0 & 0 & 0 \\
\hline $\begin{array}{c}20.6 \\
5\end{array}$ & 3-Octanol & 5,021 & 10,176 & 0 & 0 & 6,694 & 0 & 0 & 0 & 4,583 & 3,283 & 0 & 7,011 & 0 \\
\hline $\begin{array}{c}20.9 \\
5\end{array}$ & Tetradecane & 12,912 & 21,943 & 25,936 & 23,953 & 14,859 & 31,797 & 20,273 & 23,981 & 16,134 & 25,493 & 28,78 & 24,445 & 24,8 \\
\hline $\begin{array}{c}21.2 \\
5\end{array}$ & trans-p-mentha-1(7),8-dien-2-ol & 0 & 0 & 0 & 0 & 0 & 0 & 0 & 0 & 2,068 & 0 & 0 & 0 & 0 \\
\hline $\begin{array}{c}21.4 \\
3\end{array}$ & Perillene & 0 & 0 & 11,594 & 0 & 0 & 0 & 4,717 & 0 & 0 & 0 & 0 & 0 & 0 \\
\hline $\begin{array}{c}21.8 \\
4\end{array}$ & Isobutylbenzene & 0 & 15,057 & 0 & 16,845 & 0 & 0 & 47,556 & 15,861 & 20,973 & 22,514 & 32,065 & 0 & 13,615 \\
\hline $\begin{array}{c}22.1 \\
2\end{array}$ & 1,2-Oxolinalool & 6,037 & 0 & 33,755 & 0 & 80,428 & 0 & 0 & 0 & 0 & 0 & 0 & 0 & 0 \\
\hline $\begin{array}{c}22.4 \\
4\end{array}$ & (+)-(E)-Limonene oxide & 0 & 0 & 6,428 & 0 & 0 & 0 & 0 & 0 & 0 & 0 & 0 & 0 & 0 \\
\hline $\begin{array}{c}22.5 \\
6\end{array}$ & 1-Tridecene & 4,568 & 0 & 0 & 0 & 0 & 4,416 & 0 & 0 & 0 & 0 & 0 & 3,601 & 0 \\
\hline $\begin{array}{c}22.5 \\
6\end{array}$ & Undecyl pentafluoropropionate & 0 & 0 & 0 & 0 & 0 & 0 & 0 & 5,438 & 0 & 0 & 0 & 0 & 0 \\
\hline $\begin{array}{c}22.5 \\
7\end{array}$ & 1,16-Dichlorohexadecane & 0 & 0 & 0 & 5,887 & 0 & 0 & 0 & 0 & 0 & 0 & 0 & 0 & 6,037 \\
\hline $\begin{array}{c}22.6 \\
1\end{array}$ & $\alpha$-Cubebene & 0 & 4,186 & 0 & 0 & 0 & 0 & 16,334 & 0 & 28,967 & 0 & 23,464 & 0 & 0 \\
\hline $\begin{array}{c}22.6 \\
5\end{array}$ & 1-Chloroeicosane & 0 & 9,82 & 0 & 0 & 0 & 0 & 0 & 0 & 0 & 0 & 0 & 0 & 0 \\
\hline $\begin{array}{c}22.6 \\
8\end{array}$ & 1-Tetradecene & 0 & 0 & 0 & 0 & 3,353 & 0 & 0 & 0 & 0 & 0 & 0 & 0 & 0 \\
\hline $\begin{array}{c}22.7 \\
7\end{array}$ & $\begin{array}{l}\text { Bicyclo[3.2.0]hept-2-ene, exo-4- } \\
\text { tertbutoxy- }\end{array}$ & 0 & 0 & 0 & 0 & 0 & 0 & 0 & 0 & 0 & 15,853 & 0 & 0 & 0 \\
\hline $\begin{array}{c}22.9 \\
8\end{array}$ & Methyl undecyl ether & 0 & 0 & 0 & 0 & 0 & 2,203 & 0 & 0 & 0 & 0 & 0 & 0 & 0 \\
\hline $\begin{array}{c}23.5 \\
0\end{array}$ & $\begin{array}{c}\text { Polypropylene Glycol Methyl Eth } \\
\text { er }\end{array}$ & 799,489 & 1599,327 & 1654,054 & 178,829 & 765,18 & 833,419 & 0 & 172,517 & $\begin{array}{r}1520,08 \\
6\end{array}$ & $\begin{array}{r}1615,99 \\
4\end{array}$ & 99,289 & 910,685 & 0 \\
\hline $\begin{array}{c}24.1 \\
3\end{array}$ & 2-Ethylhexanol & 719,286 & 187,687 & 0 & $\begin{array}{r}1385,67 \\
9\end{array}$ & 0 & 830,527 & $\begin{array}{r}1398,3 \\
59\end{array}$ & 1417,18 & 147,552 & 797,095 & 0 & 867,047 & $\begin{array}{r}1505,3 \\
55\end{array}$ \\
\hline $\begin{array}{c}24.3 \\
9\end{array}$ & Camphor & 0 & 0 & 0 & 0 & 1100,23 & 0 & 0 & 0 & 0 & 0 & 2232,84 & 0 & 0 \\
\hline $\begin{array}{c}24.4 \\
9\end{array}$ & Pentadecane & 0 & 17,365 & 7,446 & 0 & 0 & 20,603 & 40,721 & 0 & 15,392 & 0 & 0 & 20,415 & 0 \\
\hline $\begin{array}{c}24.5 \\
1\end{array}$ & $\begin{array}{l}\text { 2-Octen-1-ol, 3,7-dimethyl-, } \\
\text { isobutyrate, (Z)- }\end{array}$ & 0 & 0 & 0 & 0 & 0 & 0 & 0 & 0 & 0 & 25,802 & 0 & 0 & 0 \\
\hline $\begin{array}{c}24.8 \\
3\end{array}$ & Benzaldehyde & 0 & 13,513 & 0 & 0 & 0 & 0 & 0 & 0 & 0 & 0 & 0 & 0 & 0 \\
\hline $\begin{array}{c}25.3 \\
8\end{array}$ & $\begin{array}{c}\text { Methyl 2,6-dideoxy- } \alpha \text {-d-lyxo- } \\
\text { hexopyranoside }\end{array}$ & 830,201 & 920,868 & 912,518 & 776,097 & 811,795 & 885,574 & $\begin{array}{r}790,82 \\
9\end{array}$ & 789,657 & 881,883 & 878,431 & 843,065 & 974,78 & $\begin{array}{r}827,05 \\
5\end{array}$ \\
\hline
\end{tabular}


5 of 19

\begin{tabular}{|c|c|c|c|c|c|c|c|c|c|c|c|c|c|c|}
\hline $\begin{array}{c}26.0 \\
6\end{array}$ & Linalool & 64,433 & 148,032 & 0 & 0 & 1344,423 & 0 & 0 & 0 & 0 & 135,11 & 0 & 0 & $\begin{array}{r}290,59 \\
6\end{array}$ \\
\hline $\begin{array}{c}26.2 \\
3\end{array}$ & $\beta$-Curcumene & 0 & 0 & 0 & 0 & 0 & 0 & $\begin{array}{r}551,43 \\
7\end{array}$ & 0 & 0 & 0 & 0 & 0 & 0 \\
\hline $\begin{array}{c}26.3 \\
5\end{array}$ & 1-Octanol & 197,736 & 232,05 & 674,431 & 186,808 & 0 & 237,314 & $\begin{array}{r}479,07 \\
9\end{array}$ & 333,668 & 211,771 & 230,618 & 275,306 & 242,376 & $\begin{array}{r}249,61 \\
2\end{array}$ \\
\hline $\begin{array}{c}26.6 \\
3\end{array}$ & $\begin{array}{c}\text { Dipropylene glycol monomethyl } \\
\text { ether }\end{array}$ & 54,813 & 68,104 & 0 & 65,324 & 0 & 63,566 & 85,244 & 63,202 & 62,043 & 69,426 & 0 & 71,982 & 0 \\
\hline $\begin{array}{c}27.0 \\
5\end{array}$ & Caryophyllene & 0 & 626,233 & 7,951 & 37,152 & 1,13 & 72,023 & $\begin{array}{r}143,93 \\
7\end{array}$ & 38,026 & 89,446 & 224,473 & 0 & 27,025 & $\begin{array}{r}155,38 \\
8\end{array}$ \\
\hline $\begin{array}{c}27.4 \\
9\end{array}$ & 4-Carvomenthenol & 23,711 & 39,256 & 44,759 & 35,96 & 5,478 & 4,096 & 0 & 35,202 & 224,368 & 101,313 & 532,642 & 0 & 9,8 \\
\hline $\begin{array}{c}27.6 \\
1\end{array}$ & Hexadecane & 6,28 & 0 & 0 & 0 & 0 & 11,766 & 0 & 0 & 0 & 0 & 0 & 0 & 0 \\
\hline $\begin{array}{c}27.6 \\
7\end{array}$ & 1-Chloro-Heptacosane & 0 & 6,342 & 0 & 0 & 0 & 0 & 0 & 0 & 0 & 0 & 0 & 0 & 0 \\
\hline $\begin{array}{c}27.8 \\
4\end{array}$ & Ethylene glycol monohexyl ether & 0 & 29,913 & 0 & 0 & 0 & 29,709 & 0 & 0 & 0 & 0 & 0 & 0 & 0 \\
\hline $\begin{array}{c}27.8 \\
6\end{array}$ & $\begin{array}{c}\mathrm{N}-[2-[\mathrm{p}- \\
\text { Methoxybenzyl]amino]ethylazirid } \\
\text { ine }\end{array}$ & 40,312 & 0 & 0 & 0 & 0 & 0 & 0 & 0 & 0 & 0 & 0 & 0 & 0 \\
\hline $\begin{array}{c}28.0 \\
5\end{array}$ & $\begin{array}{l}\text { 1-(4-piperidinylcarbonyl) } \\
\text { piperidine }\end{array}$ & 153,789 & 161,876 & 209,939 & 184,506 & 201,02 & 204,666 & $\begin{array}{r}266,74 \\
3\end{array}$ & 221,021 & 200,268 & 182,797 & 214,645 & 234,7 & $\begin{array}{r}193,11 \\
6\end{array}$ \\
\hline $\begin{array}{c}28.3 \\
3\end{array}$ & cis-p-Menth-2-en-1-ol & 0 & 0 & 0 & 0 & 0 & 0 & 0 & 0 & 3,932 & 0 & 0 & 0 & 0 \\
\hline $\begin{array}{c}28.4 \\
6\end{array}$ & Dihydro carveol & 0 & 0 & 0 & 0 & 0 & 0 & 0 & 0 & 0 & 0 & 0 & 0 & 2,741 \\
\hline $\begin{array}{c}28.4 \\
7\end{array}$ & trans- $\beta$-Terpineol & 0 & 0 & 0 & 25,333 & 0 & 0 & 0 & 22,948 & 0 & 0 & 0 & 0 & 0 \\
\hline $\begin{array}{c}28.5 \\
1\end{array}$ & Aromandendrene & 0 & 7,305 & 0 & 0 & 0 & 0 & 0 & 0 & 0 & 0 & 0 & 0 & 0 \\
\hline $\begin{array}{c}28.5 \\
5\end{array}$ & $\gamma$-Elemene & 0 & 0 & 0 & 0 & 0 & 0 & 59,116 & 0 & 0 & 0 & 0 & 0 & 0 \\
\hline $\begin{array}{c}28.6 \\
6\end{array}$ & Humulen-(v1) & 0 & 0 & 0 & 0 & 0 & 0 & 0 & 0 & 0 & 0 & 3,398 & 0 & 0 \\
\hline $\begin{array}{c}28.6 \\
9\end{array}$ & Menthol & 0 & 0 & 0 & 0 & 0 & 1,365 & 0 & 0 & 0,968 & 0 & 0 & 0 & 0 \\
\hline $\begin{array}{c}28.8 \\
8\end{array}$ & 5-Benzoylpentanoic acid & 0 & 3,874 & 0 & 0 & 0 & 0 & 0 & 0 & 0 & 0 & 0 & 0 & 0 \\
\hline $\begin{array}{c}28.9 \\
5\end{array}$ & $\alpha$-Terpinyl acetate & 0 & 0 & 0 & 0 & 0 & 0 & 0 & 1,306 & 0 & 0 & 0 & 0 & 0 \\
\hline $\begin{array}{c}29.0 \\
1\end{array}$ & 1-Octadecene & 0 & 0 & 0 & 0 & 0 & 1,641 & 0 & 0 & 0 & 0 & 0 & 0 & 0 \\
\hline $\begin{array}{c}29.0 \\
5\end{array}$ & $\beta$-copaene & 0 & 0 & 0 & 0 & 0 & 0 & 0 & 0 & 2,428 & 0 & 0 & 0 & 0 \\
\hline $\begin{array}{c}29.0 \\
8\end{array}$ & Pinocarveol & 0 & 0 & 6,809 & 0 & 0 & 0 & 0 & 0 & 0 & 0 & 0 & 0 & 0 \\
\hline $\begin{array}{c}29.1 \\
1\end{array}$ & 1-Heptadecene & 0 & 0 & 0 & 2,04 & 0 & 0 & 0 & 0 & 0 & 0 & 0 & 0 & 0 \\
\hline $\begin{array}{c}29.2 \\
4\end{array}$ & Humulene & 0 & 128,225 & 0 & 0 & 0 & 8,32 & 48,543 & 0 & 9,174 & 1,697 & 73,704 & 0 & 0 \\
\hline $\begin{array}{c}29.5 \\
2\end{array}$ & $\begin{array}{c}\text { 2-(3,4-Dibromo-4- } \\
\text { methylcyclohexyl)propan-2-ol }\end{array}$ & 0 & 0 & 0 & 0 & 0 & 0 & 0 & 0 & 0 & 0 & 0 & 0 & 42,658 \\
\hline $\begin{array}{c}29.5 \\
4\end{array}$ & Dipropylene glycol, butyl ether & 0 & 15,077 & 23,058 & 0 & 7,918 & 0 & 0 & 0 & 13,056 & 12,683 & 0 & 0 & 0 \\
\hline $\begin{array}{c}29.5 \\
7\end{array}$ & Anethole & 38,326 & 0 & 0 & 43,056 & 0 & 26,319 & 0 & 81,61 & 0 & 0 & 0 & 18,306 & 0 \\
\hline $\begin{array}{c}29.8 \\
7\end{array}$ & 3,3'-Oxybis-2-butanol & 0 & 0 & 0 & 0 & 5,383 & 0 & 0 & 0 & 0 & 0 & 0 & 0 & 5,289 \\
\hline $\begin{array}{c}29.8 \\
8\end{array}$ & 3-Chloro-2-methyl-2-pentanol & 0 & 0 & 12,297 & 0 & 0 & 0 & 0 & 0 & 0 & 0 & 0 & 0 & 0 \\
\hline $\begin{array}{c}29.9 \\
8\end{array}$ & $\gamma$-Muurolene & 0 & 12,456 & 0 & 0 & 0 & 0 & 71,655 & 0 & 3,273 & 0 & 85,262 & 0 & 0 \\
\hline $\begin{array}{c}29.9 \\
8\end{array}$ & $\begin{array}{c}\text { 1-[1-Methyl-2-(2- } \\
\text { propenyloxy)ethoxy]-2-propanol }\end{array}$ & 0 & 0 & 0 & 19,985 & 6,765 & 25,422 & 0 & 0 & 27,525 & 17,597 & 0 & 7,386 & 7,917 \\
\hline $\begin{array}{c}30.0 \\
1\end{array}$ & Myrcenol & 0 & 0 & 0 & 0 & 0 & 0 & 0 & 20,922 & 0 & 0 & 0 & 0 & 0 \\
\hline
\end{tabular}


6 of 19

\begin{tabular}{|c|c|c|c|c|c|c|c|c|c|c|c|c|c|c|}
\hline $\begin{array}{c}30.1 \\
3\end{array}$ & Isoledene & 0 & 50,409 & 0 & 0 & 0 & 0 & 0 & 0 & 0 & 0 & 0 & 0 & 0 \\
\hline $\begin{array}{c}30.2 \\
9\end{array}$ & Estragole & $\begin{array}{r}2018,73 \\
8\end{array}$ & 0 & 0 & 0 & 0 & 0 & 0 & 0 & 0 & 0 & 0 & 0 & 0 \\
\hline $\begin{array}{c}30.4 \\
3\end{array}$ & L- $\alpha$-Terpineol & 63,995 & 21,515 & 883,389 & 701,913 & 42,932 & 3,479 & 0 & 723,138 & 25,325 & 46,623 & 178,197 & 0 & 73,644 \\
\hline $\begin{array}{c}30.6 \\
4\end{array}$ & Heptadecane & 0 & 0 & 0 & 0 & 0 & 9,116 & 0 & 0 & 10,016 & 0 & 0 & 9,96 & 0 \\
\hline $\begin{array}{c}30.7 \\
7\end{array}$ & $\begin{array}{c}\text { Pseduosarsasapogenin-5,20-dien } \\
\text { methyl ether }\end{array}$ & 0 & 14,329 & 0 & 0 & 0 & 0 & 0 & 0 & 0 & 0 & 0 & 0 & 0 \\
\hline $\begin{array}{c}30.7 \\
9\end{array}$ & Longifolene-(V4) & 0 & 0 & 0 & 0 & 0 & 0 & 52,216 & 0 & 0 & 0 & 0 & 0 & 0 \\
\hline $\begin{array}{c}30.9 \\
6\end{array}$ & $\gamma$-Neoclovene & 0 & 0 & 46,115 & 0 & 0 & 0 & 0 & 0 & 0 & 0 & 14,805 & 0 & 0 \\
\hline $\begin{array}{c}31.0 \\
3\end{array}$ & 1,2-Nonadecanediol & 0 & 0 & 0 & 0 & 0 & 1,122 & 0 & 0 & 0 & 0 & 0 & 0 & 0 \\
\hline $\begin{array}{c}31.1 \\
1\end{array}$ & Pentamethylcyclopentadiene & 0 & 0 & 0 & 0 & 0 & 0 & 69,26 & 0 & 0 & 0 & 0 & 0 & 0 \\
\hline $\begin{array}{c}31.2 \\
3\end{array}$ & $\beta$-Bisabolene & 0 & 0 & 0 & 0 & 0 & 0 & 0 & 0 & 5,54 & 7,697 & 9,478 & 0 & 0 \\
\hline $\begin{array}{c}31.2 \\
5\end{array}$ & 2-Undecanol & 0 & 1,232 & 1,184 & 0 & 0 & 0,805 & 0 & 0 & 0 & 0 & 0 & 0 & 0,115 \\
\hline $\begin{array}{c}31.3 \\
4\end{array}$ & $\gamma$-Elemene & 0 & 0 & 0 & 5,714 & 0 & 0 & 0 & 5,469 & 0 & 0 & 0 & 0 & 0 \\
\hline $\begin{array}{c}31.4 \\
1\end{array}$ & $\begin{array}{l}\text { 3-chlorophenyl 2-methoxyethyl } \\
\text { ester carbonic acid }\end{array}$ & 0 & 0 & 0 & 0 & 0 & 0,646 & 0 & 0 & 0 & 0 & 0 & 0 & 0 \\
\hline $\begin{array}{c}31.6 \\
0\end{array}$ & [4.2.2]Propella-2,4,7,9-tetraene & 4,882 & 0 & 0 & 0 & 0 & 0 & 0 & 0 & 0 & 0 & 0 & 0 & 0 \\
\hline $\begin{array}{c}31.7 \\
4\end{array}$ & $\begin{array}{l}\text { Ethyl 2-(5-methyl-5- } \\
\text { vinyltetrahydrofuran-2- } \\
\text { yl)propan-2-yl carbonate }\end{array}$ & 0 & 0 & 0 & 0 & 5,552 & 0 & 0 & 0 & 0 & 0 & 0 & 0 & 0 \\
\hline $\begin{array}{c}31.7 \\
7\end{array}$ & 1-Azepan-1-yl-2-bromethanone & 0 & 0 & 0 & 0 & 0 & 1,943 & 0 & 0 & 3,241 & 0 & 0 & 0 & 0 \\
\hline $\begin{array}{c}32.0 \\
6\end{array}$ & $\beta$-Cadinene & 0 & 53,199 & 0 & 0 & 0 & 0 & 96,048 & 0 & 30,06 & 0 & 51,703 & 0 & 0 \\
\hline $\begin{array}{c}32.0 \\
9\end{array}$ & Octyl ether & 0 & 0 & 35,804 & 14,093 & 11,88 & 17,81 & 0 & 17,125 & 0 & 12,27 & 0 & 13,899 & 14,442 \\
\hline $\begin{array}{c}32.5 \\
3\end{array}$ & Nonyl-cyclopropane & 138,586 & 165,025 & 155,907 & 130,933 & 136,659 & 173,198 & $\begin{array}{r}151,35 \\
8\end{array}$ & 133,82 & 147,478 & 130,037 & 149,199 & 155,748 & $\begin{array}{r}127,86 \\
7\end{array}$ \\
\hline $\begin{array}{c}32.9 \\
9\end{array}$ & $\alpha$-Cadinene & 0 & 2,833 & 0 & 0 & 0 & 0 & 8,654 & 0 & 0 & 0 & 0 & 0 & 0 \\
\hline $\begin{array}{c}33.2 \\
2\end{array}$ & 2-Butyloctanol & 0 & 0 & 29,365 & 0 & 8,386 & 0 & 0 & 0 & 0 & 0 & 10,621 & 0 & 0 \\
\hline $\begin{array}{c}33.2 \\
3\end{array}$ & 2-methyldecan-1-ol & 57,986 & 80,795 & 15,622 & 79,421 & 26,102 & 76,32 & 6,351 & 74,938 & 60,145 & 44,958 & 28,523 & 31,801 & 6,512 \\
\hline $\begin{array}{c}33.4 \\
1\end{array}$ & Isopinocarveol & 0 & 0 & 0 & 0 & 0 & 0 & 15,134 & 0 & 0 & 0 & 0 & 0 & 0 \\
\hline $\begin{array}{c}33.5 \\
5\end{array}$ & (S)-2-Methyl-1-dodecanol & 0 & 0 & 0 & 0 & 0 & 5,483 & 0 & 46,717 & 5,156 & 0 & 0 & 0 & 0 \\
\hline $\begin{array}{c}33.6 \\
0\end{array}$ & 1-Undecanol & 0 & 0 & 0 & 0 & 0 & 0 & 0 & 5,534 & 0 & 0 & 0 & 0 & 0 \\
\hline $\begin{array}{c}34.0 \\
4\end{array}$ & $\begin{array}{c}\text { Ethylene glycol monododecyl } \\
\text { ether }\end{array}$ & 0 & 18,094 & 12,665 & 15,443 & 10,189 & 18,65 & 22,399 & 19,812 & 13,57 & 9,756 & 16,331 & 14,55 & 12,93 \\
\hline $\begin{array}{c}34.1 \\
1\end{array}$ & trans-calamenene & 0 & 0 & 0 & 0 & 0 & 0 & 20,503 & 0 & 0 & 0 & 0 & 0 & 0 \\
\hline $\begin{array}{c}34.4 \\
6\end{array}$ & trans-3-Caren-2-ol & 0 & 0 & 6,559 & 0 & 1,213 & 0 & 0 & 0 & 0 & 0 & 0 & 0 & 0 \\
\hline $\begin{array}{c}34.8 \\
8\end{array}$ & Thymol & 0 & 0 & 0 & 8,756 & 0 & 0 & 0 & 3,807 & 0 & 86,856 & 0 & 0 & 87,723 \\
\hline $\begin{array}{c}34.8 \\
8\end{array}$ & Geraniol & 0 & 0 & 24,141 & 0 & 57,715 & 0 & 0 & 0 & 3,016 & 3,344 & 0 & 0 & 0 \\
\hline $\begin{array}{c}34.9 \\
4\end{array}$ & $\begin{array}{l}\text { Tridecyl adamantane-1- } \\
\text { carboxylate }\end{array}$ & 0 & 1,678 & 0 & 0 & 0 & 0 & 0 & 0 & 0 & 0 & 0 & 0 & 0 \\
\hline $\begin{array}{c}35.3 \\
3\end{array}$ & E-11,13-Tetradecadien-1-ol & 5,544 & 0 & 0 & 0 & 6,806 & 0 & 6,868 & 0 & 0 & 6,215 & 0 & 0 & 0 \\
\hline $\begin{array}{c}35.4 \\
4\end{array}$ & $\begin{array}{l}\text { N-Methyl-N-(2- } \\
\text { ethylhexyl)trifluoroacetamide }\end{array}$ & 0 & 0 & 0 & 0 & 0 & 9,735 & 0 & 6,922 & 0 & 0 & 0 & 0 & 0 \\
\hline
\end{tabular}


7 of 19

\begin{tabular}{|c|c|c|c|c|c|c|c|c|c|c|c|c|c|c|}
\hline $\begin{array}{c}35.4 \\
5\end{array}$ & Safrole & 0 & 248,239 & 0 & 0 & 0 & 0 & 0 & 0 & 99,36 & 0 & 28,938 & 0 & 0 \\
\hline $\begin{array}{c}35.4 \\
5\end{array}$ & $\begin{array}{l}\text { 1-(2,6-Dimethylmorpholin-4-yl)-2- } \\
\text { ethyl-hexan-1-one }\end{array}$ & 0 & 0 & 0 & 0 & 0 & 0 & 0 & 0 & 0 & 0 & 0 & 12,516 & 0 \\
\hline $\begin{array}{c}36.4 \\
3\end{array}$ & $\alpha$-Calacorene & 0 & 3,395 & 0 & 0 & 0 & 0 & 0 & 0 & 0 & 0 & 0 & 0 & 0 \\
\hline $\begin{array}{c}36.7 \\
1\end{array}$ & $\begin{array}{l}\text { N-(2-Furanylmethylene)-3- } \\
\text { methyl-1-butanamine }\end{array}$ & 0 & 0 & 0 & 0 & 0 & 1,756 & 0 & 0 & 0 & 0 & 0 & 0 & 0 \\
\hline $\begin{array}{c}37.5 \\
2\end{array}$ & Caryophyllene oxide & 0 & 40,715 & 0 & 0 & 0 & 0 & 0 & 0 & 0 & 124,189 & 0 & 0 & 29,331 \\
\hline $\begin{array}{c}37.5 \\
5\end{array}$ & Decyl ether & 0 & 9,045 & 0 & 0 & 0 & 5,414 & 0 & 0 & 4,988 & 4,558 & 0 & 0 & 0 \\
\hline $\begin{array}{c}37.6 \\
7\end{array}$ & $\begin{array}{l}\text { 2,6-Dimethyl-3,7-octadiene-2,6- } \\
\text { diol }\end{array}$ & 0 & 0 & 25,187 & 0 & 16,676 & 0 & 0 & 0 & 0 & 0 & 0 & 0 & 0 \\
\hline $\begin{array}{c}37.6 \\
7\end{array}$ & $\begin{array}{l}\text { 1-Ethyl-2,3-dimethyl-piperidin-4- } \\
\text { one }\end{array}$ & 0 & 0 & 0 & 0 & 0 & 0 & 20,108 & 0 & 0 & 0 & 0 & 0 & 0 \\
\hline $\begin{array}{c}37.8 \\
1\end{array}$ & $\begin{array}{l}\text { N-[4-Bromo-n-butyl]-2- } \\
\text { piperidinone }\end{array}$ & 0 & 0 & 0 & 0 & 0 & 0 & 0 & 0 & 0 & 17,168 & 0 & 0 & 0 \\
\hline $\begin{array}{c}38.0 \\
9\end{array}$ & 1-Dodecanol & 7,115 & 0 & 0 & 9,707 & 8,989 & 12,267 & 9,936 & 6,38 & 7,658 & 0 & 33,877 & 0 & 5,598 \\
\hline $\begin{array}{c}38.4 \\
1\end{array}$ & Limonene glycol & 0 & 0 & 16,8 & 0 & 0 & 0 & 0 & 0 & 0 & 0 & 0 & 0 & 0 \\
\hline $\begin{array}{c}38.5 \\
4\end{array}$ & $\begin{array}{c}(2 \alpha, 3 \alpha, 5 \beta)-1,1,2 \text {-Trimethyl-3,5- } \\
\text { bis(1-methylethenyl)-cyclohexane }\end{array}$ & 0 & 0 & 0 & 0 & 0 & 0 & 0 & 0 & 0 & 0,921 & 0 & 0 & 0 \\
\hline $\begin{array}{c}38.8 \\
3\end{array}$ & Diethylene glycol hexyl ether & 3,59 & 5,71 & 9,887 & 4,689 & 4,286 & 3,346 & 4,14 & 3,583 & 2,828 & 4,056 & 4,905 & 2,872 & 2,959 \\
\hline $\begin{array}{c}39.2 \\
7\end{array}$ & $\begin{array}{l}\text { Benzenepropanoic acid, } \beta \text {-[(tert- } \\
\text { butyldimethylsilyl)oxy]-, tert- } \\
\text { butyldimethylsilyl ester }\end{array}$ & 0 & 0 & 0 & 0 & 0 & 1,672 & 0 & 0 & 0 & 0 & 0 & 0 & 0 \\
\hline $\begin{array}{c}39.2 \\
8\end{array}$ & 2-Allyl-1,4-dimethoxybenzene & 0 & 4,292 & 0 & 0 & 0 & 0 & 0 & 0 & 13,476 & 0 & 3,852 & 0 & 0 \\
\hline $\begin{array}{c}39.3 \\
0\end{array}$ & P-Anisaldehyde & 2,4 & 0 & 0 & 0 & 0 & 0 & 0 & 0 & 0 & 0 & 0 & 0 & 0 \\
\hline $\begin{array}{c}39.5 \\
0\end{array}$ & Humulene epoxide II & 0 & 9,314 & 0 & 0 & 0 & 0,527 & 0 & 0 & 0 & 0 & 0 & 0 & 0 \\
\hline $\begin{array}{c}39.6 \\
3\end{array}$ & Isosafrol & 0 & 0 & 0 & 0 & 0 & 0 & 0 & 0 & 0,962 & 0 & 0 & 0 & 0 \\
\hline $\begin{array}{c}39.9 \\
2\end{array}$ & Benzylidene-hexyl-amine & 0 & 146,116 & 0 & 0 & 2,485 & 1,319 & 0 & 0 & 0 & 0,475 & 10,762 & 0 & 0 \\
\hline $\begin{array}{c}40.0 \\
4\end{array}$ & Nerolidol & 0 & 0 & 28,949 & 0 & 0 & 0 & 3,096 & 0 & 0 & 0 & 0 & 0 & 0 \\
\hline $\begin{array}{c}40.8 \\
6\end{array}$ & Elemol & 0 & 0 & 0 & 1,638 & 0 & 0 & 6,935 & 3,05 & 1,116 & 0 & 0 & 0 & 0 \\
\hline $\begin{array}{c}41.0 \\
9\end{array}$ & Guaiol & 0 & 0 & 0 & 0 & 0 & 0 & 0 & 0 & 1,202 & 0 & 0 & 0 & 0 \\
\hline $\begin{array}{c}41.2 \\
4\end{array}$ & p-Menth-2-en-1,4-diol & 0 & 0 & 0 & 0 & 0 & 0 & 0 & 0 & 0 & 0 & 4,999 & 0 & 0 \\
\hline $\begin{array}{c}41.5 \\
1\end{array}$ & Dehydroterpin Monoacetate & 0 & 0 & 3,902 & 0 & 0 & 0 & 0 & 0 & 0 & 0 & 0 & 0 & 0 \\
\hline $\begin{array}{c}41.5 \\
7\end{array}$ & Cedrol & 0 & 0 & 0 & 0 & 0 & 0 & $\begin{array}{r}125,83 \\
1\end{array}$ & 0 & 1,326 & 0,981 & 0 & 0 & 0 \\
\hline $\begin{array}{c}41.6 \\
7\end{array}$ & Acetocinnamone & 0 & 2,301 & 0 & 0 & 0 & 0 & 0 & 0 & 0 & 0 & 0 & 0 & 0 \\
\hline $\begin{array}{c}41.6 \\
9\end{array}$ & p-Propylguaiacol & 0 & 0 & 0 & 0 & 0 & 0,193 & 0 & 0 & 0 & 0 & 0 & 0 & 0 \\
\hline $\begin{array}{c}41.8 \\
3\end{array}$ & Spathulenol & 0 & 2,182 & 0 & 0 & 0 & 0 & 0 & 3,751 & 0,404 & 0 & 0 & 0 & 0 \\
\hline $\begin{array}{c}41.9 \\
4\end{array}$ & $\begin{array}{l}\text { 3-Acetoxy-4-(1-hydroxy-1- } \\
\text { methylethyl)-1-methyl- } \\
\text { cyclohexene }\end{array}$ & 0 & 0 & 26,72 & 6,871 & 0 & 0 & 0 & 0 & 0 & 0 & 0 & 0 & 0 \\
\hline $\begin{array}{c}42.3 \\
0\end{array}$ & $\begin{array}{c}\text { 2,6-Dimethyl-1,7-octadiene-3,6- } \\
\text { diol }\end{array}$ & 0 & 0 & 3,248 & 0 & 2,705 & 0 & 0 & 0 & 0 & 0 & 0 & 0 & 0 \\
\hline $\begin{array}{c}42.5 \\
5\end{array}$ & Longifolene-I2 & 0 & 0 & 0 & 0 & 0 & 0 & 5,791 & 0 & 0 & 0 & 0 & 0 & 0 \\
\hline $\begin{array}{c}42.7 \\
4\end{array}$ & Cinnamyl ester acetic acid & 0 & 25,656 & 0 & 0 & 0 & 0 & 0 & 0 & 0 & 0 & 0 & 0 & 0 \\
\hline $\begin{array}{c}42.7 \\
9\end{array}$ & 4-Methyl-3-heptanol & 3,354 & 0 & 4,683 & 4,549 & 3,469 & 1,271 & 0 & 5,57 & 2,697 & 4,791 & 3,549 & 2,748 & 2,838 \\
\hline
\end{tabular}


8 of 19

\begin{tabular}{|c|c|c|c|c|c|c|c|c|c|c|c|c|c|c|}
\hline $\begin{array}{c}43.0 \\
6\end{array}$ & Chavibetol & 0 & 246,972 & 8,34 & 0 & 0 & 212,738 & 9,924 & 0 & 3,412 & 0 & 13,884 & 12,511 & 6,1 \\
\hline $\begin{array}{c}43.2 \\
5\end{array}$ & Myristyl alcohol & 1,32 & 0 & 3,321 & 2,18 & 0 & 0 & 0 & 0 & 0 & 0 & 0 & 1,975 & 1,737 \\
\hline $\begin{array}{c}43.3 \\
7\end{array}$ & $\begin{array}{l}\text { 1-(Isooctyloxy)-2-methyl-2- } \\
\text { propanol }\end{array}$ & 1,659 & 6,09 & 2,954 & 0 & 0 & 7,028 & 0 & 0 & 4,578 & 0 & 2,653 & 2,511 & 0 \\
\hline $\begin{array}{c}43.4 \\
4\end{array}$ & $\alpha$-Cadinol & 0 & 0 & 0 & 0 & 0 & 0 & 67,701 & 0 & 0 & 0 & 0 & 0 & 0 \\
\hline $\begin{array}{c}43.4 \\
8\end{array}$ & Isomethyleugenol & 0 & 0 & 0 & 0 & 0 & 0 & 0 & 0 & 1,457 & 0 & 0 & 0 & 0 \\
\hline $\begin{array}{c}43.7 \\
1\end{array}$ & Carvacrol & 5,515 & 0 & 0 & 1,916 & 0 & 2,1 & 0 & 11,253 & 0,686 & 191,278 & 0 & 0,634 & 31,214 \\
\hline $\begin{array}{c}43.9 \\
0\end{array}$ & Geranyllinalool & 0 & 0 & 0 & 0 & 0 & 0 & 12,581 & 0 & 0 & 0 & 0 & 0 & 0 \\
\hline $\begin{array}{c}44.0 \\
0\end{array}$ & $\begin{array}{l}\text { Diethylene glycol monododecyl } \\
\text { ether }\end{array}$ & 1,216 & 2,494 & 0 & 2,195 & 1,563 & 1,726 & 0 & 0 & 0 & 1,199 & 0 & 1,782 & 0 \\
\hline $\begin{array}{c}44.4 \\
7\end{array}$ & Hedycaryol & 0 & 0 & 0 & 0 & 0 & 0 & 3,761 & 0 & 0 & 0 & 0 & 0 & 0 \\
\hline $\begin{array}{c}44.5 \\
6\end{array}$ & Asarone & 0 & 0 & 0 & 0 & 0 & 0 & 0 & 0 & 36,598 & 0 & 0 & 0 & 0 \\
\hline $\begin{array}{c}44.9 \\
6\end{array}$ & cis-sesquisabinene hydrate & 0 & 0 & 0 & 0 & 0 & 0 & 0 & 0 & 2,327 & 0 & 0 & 0 & 0 \\
\hline $\begin{array}{c}45.0 \\
1\end{array}$ & geranyl- $\alpha$-terpinene & 0 & 0 & 0 & 0 & 0 & 0 & 11,772 & 0 & 0 & 0 & 0 & 0 & 0 \\
\hline $\begin{array}{c}45.3 \\
2\end{array}$ & Myristicin & 0 & 5,066 & 6,729 & 0 & 0 & 0 & 0 & 0 & 69,475 & 0,532 & 0 & 0 & 0 \\
\hline $\begin{array}{c}45.8 \\
0\end{array}$ & Cinnamyl alcohol & 0 & 10,64 & 0 & 0 & 0 & 0 & 0 & 0 & 0 & 0 & 0 & 0 & 0 \\
\hline $\begin{array}{c}45.8 \\
3\end{array}$ & Benzylidene-hexyl-amine & 0 & 0 & 0 & 0 & 0 & 0 & 0 & 0 & 0,656 & 0 & 0 & 0 & 0 \\
\hline $\begin{array}{c}45.8 \\
7\end{array}$ & $\begin{array}{l}\text { 6-Isopropenyl-4,8a-dimethyl- } \\
\text { 1,2,3,5,6,7,8,8a-octahydro- } \\
\text { naphthalen-2-ol }\end{array}$ & 0 & 0 & 0 & 0 & 0 & 0 & 10,839 & 0 & 0 & 3,595 & 0 & 0 & 0 \\
\hline $\begin{array}{c}45.9 \\
1\end{array}$ & Verbenol & 0 & 0 & 6,171 & 0 & 0 & 0 & 0 & 0 & 0 & 3,081 & 0 & 0 & 0 \\
\hline $\begin{array}{c}45.9 \\
7\end{array}$ & $\begin{array}{c}\text { bis(dimethylhydrazone)ethanedia } \\
1\end{array}$ & 0 & 0 & 0 & 0 & 0 & 0 & 0 & 0 & 0 & 0 & 0 & 1,693 & 0 \\
\hline $\begin{array}{c}46.5 \\
0\end{array}$ & Sclarene & 0 & 0 & 0 & 0 & 0 & 0 & 6,303 & 0 & 0 & 0 & 0 & 0 & 0 \\
\hline $\begin{array}{c}46.6 \\
7\end{array}$ & Hydroxy- $\alpha$-terpenyl acetate & 0 & 0 & 19,031 & 2,294 & 0 & 0 & 0 & 2,741 & 0 & 1,691 & 0 & 3,428 & 0 \\
\hline $\begin{array}{c}46.8 \\
8\end{array}$ & Manoyl oxide & 0 & 0 & 0 & 0 & 0 & 0 & 9,54 & 0 & 0 & 0 & 0 & 0 & 0 \\
\hline $\begin{array}{c}46.9 \\
5\end{array}$ & Isoaromadendrene epoxide & 0 & 2,038 & 0 & 0 & 0 & 0 & 0 & 1,167 & 0 & 15,137 & 3,242 & 0 & 0 \\
\hline $\begin{array}{c}47.1 \\
6\end{array}$ & $\begin{array}{l}\text { (2S,6R)-1-Methyl-2,6- } \\
\text { dipentylpiperidin-4-one }\end{array}$ & 0 & 1,461 & 0 & 0 & 0 & 0 & 0 & 0 & 0 & 1,057 & 0 & 0 & 0 \\
\hline $\begin{array}{c}47.4 \\
4\end{array}$ & trans-Isoeugenol & 0 & 2,544 & 0 & 0 & 0 & 2,207 & 0 & 0 & 0,396 & 0 & 1,169 & 0,896 & 0 \\
\hline $\begin{array}{c}48.0 \\
0\end{array}$ & $\alpha$-Gurjunene & 0 & 0 & 0 & 0 & 0 & 0 & 12,013 & 0 & 0 & 5,832 & 0 & 0 & 0 \\
\hline $\begin{array}{c}48.3 \\
7\end{array}$ & Terpineol & 0 & 0,928 & 12,834 & 0 & 0 & 0,542 & 0 & 1,403 & 0 & 0 & 0 & 0 & 0 \\
\hline $\begin{array}{c}50.1 \\
3\end{array}$ & Cembrene & 0 & 0 & 0 & 0 & 0 & 0 & 22,917 & 0 & 3,793 & 0 & 0 & 0 & 0 \\
\hline $\begin{array}{c}50.5 \\
9\end{array}$ & $\begin{array}{l}\text { 1,3,3-Trimethyl-6-hydroxy-2- } \\
\text { oxabicyclo[222]octan-7-one }\end{array}$ & 0 & 0 & 1,092 & 0 & 0 & 0 & 0 & 0 & 0 & 0 & 0 & 0 & 0 \\
\hline $\begin{array}{c}51.4 \\
9\end{array}$ & $\begin{array}{l}\text { 7-Isopropyl-1,1,4a-trimethyl- } \\
\text { 1,2,3,4,4a,9,10,10a- } \\
\text { octahydrophenanthrene }\end{array}$ & 0 & 0 & 0 & 0 & 0 & 0 & 7,343 & 0 & 0 & 0 & 0 & 0 & 0 \\
\hline $\begin{array}{c}51.8 \\
4\end{array}$ & $\begin{array}{l}\text { trans-Octahydro-2-(4- } \\
\text { methoxyphenyl)-2H-1,3- } \\
\text { benzoxazine }\end{array}$ & 0 & 0 & 0 & 0 & 0 & 0,75 & 0 & 0 & 0 & 0 & 0 & 0 & 0 \\
\hline $\begin{array}{c}51.8 \\
5\end{array}$ & $\begin{array}{c}5-(4- \\
\text { Methoxybenzylideneamino)penta } \\
\mathrm{n}-1-\mathrm{ol}\end{array}$ & 4,074 & 0 & 0 & 0 & 0 & 0 & 0 & 0 & 0 & 0 & 0 & 0 & 0 \\
\hline $\begin{array}{c}53.3 \\
4\end{array}$ & $\begin{array}{l}\text { 7,11-Epoxymegastigma-5(6)-en-9- } \\
\text { one }\end{array}$ & 0 & 0 & 0 & 0 & 0 & 0 & 0 & 0 & 0 & 2,928 & 0 & 0 & 0 \\
\hline
\end{tabular}




\begin{tabular}{|c|c|c|c|c|c|c|c|c|c|c|c|c|c|c|}
\hline $\begin{array}{c}53.4 \\
2\end{array}$ & $\begin{array}{l}\text { 1-Butanamine, } \mathrm{N}-(3-\text { phenyl-2- } \\
\text { propenylidene)- }\end{array}$ & 0 & 9,239 & 0 & 0 & 0 & 0 & 0 & 0 & 0 & 0 & 0 & 0 & 0 \\
\hline $\begin{array}{c}55.5 \\
3\end{array}$ & Benzyl Benzoate & 0 & 7,836 & 0 & 0 & 0 & 0 & 0 & 0 & 0 & 0 & 0 & 0 & 0 \\
\hline $\begin{array}{c}56.2 \\
7\end{array}$ & $\begin{array}{l}\text { Ethyl 14-hydroxy-3,6,9,12- } \\
\text { tetraoxatetradecan-1-oate }\end{array}$ & 0 & 0 & 0 & 0 & 0 & 0 & 0 & 0 & 0 & 0 & 0 & 1,852 & 0 \\
\hline $\begin{array}{c}58.6 \\
1\end{array}$ & Hinokitol & 2,488 & 0 & 0 & 0 & 0 & 0 & 0 & 0 & 0 & 0 & 0 & 0 & 0 \\
\hline
\end{tabular}

Starting from Table 1 listing all 204 VOCs identified in this study, most are compounds present in more than one oil. On the contrary, in Table 2, characteristic VOCs for each one of the samples are shown, sorted by decreasing abundance. After the analysis 95 compounds out of 204 were characteristic of one of the selected essential oils, this identified molecules could be used in future works as markers of the presence of a determinate essential oil. In terms of abundance the oils that present characteristics chemical compounds from higher to the lower number were ordered as: Cinnamon (15), Juniper (14), Nutmeg (12), Carnation (11), Mustard (9), Cardamom (8), Basil and Oregano (6 respectively), Niaouli and Thyme (4 respectively), Coriander (3), Rosemary (2) and Cayeput (1).

Table 2. Characteristic VOCs for each EO, sorted by decreasing abundance.

\begin{tabular}{|c|c|}
\hline EO & Characteristic VOCs \\
\hline \multirow{6}{*}{ Basil } & Estragole \\
\hline & N-[2-[p-Methoxybenzyl]amino]ethylaziridine \\
\hline & [4.2.2]Propella-2,4,7,9-tetraene \\
\hline & 5-(4-Methoxybenzylideneamino)pentan-1-ol \\
\hline & Hinokitol \\
\hline & P-Anisaldehyde \\
\hline \multirow{15}{*}{ Cinnamon } & Isoledene \\
\hline & Cinnamyl ester acetic acid \\
\hline & N-[(4-Hydroxy)hydrocinnamoyl]-Benzeneethanamine \\
\hline & Pseduosarsasapogenin-5,20-dien methyl ether \\
\hline & Benzaldehyde \\
\hline & Cinnamyl alcohol \\
\hline & 1-Chloroeicosane \\
\hline & 1-Butanamine, N-(3-phenyl-2-propenylidene)- \\
\hline & Benzyl Benzoate \\
\hline & Aromandendrene \\
\hline & 1-Chloro-Heptacosane \\
\hline & 5-Benzoylpentanoic acid \\
\hline & $\alpha$-Calacorene \\
\hline & Acetocinnamone \\
\hline & Tridecyl adamantane-1-carboxylate \\
\hline \multirow{5}{*}{ Cardamom } & Methyl Heptenone \\
\hline & Limonene glycol \\
\hline & 3-Chloro-2-methyl-2-pentanol \\
\hline & Pinocarveol \\
\hline & $(+)-($ E)-Limonene oxide \\
\hline
\end{tabular}




\begin{tabular}{|c|c|}
\hline & Methoxyethylene \\
\hline & Dehydroterpin Monoacetate \\
\hline & 1,3,3-Trimethyl-6-hydroxy-2-oxabicyclo[222]octan-7-one \\
\hline Cayeput & 1-Heptadecene \\
\hline \multirow{3}{*}{ Coriander } & Ethyl 2-(5-methyl-5-vinyltetrahydrofuran-2-yl)propan-2-yl carbonate \\
\hline & 1-Tetradecene \\
\hline & N,N-Dimethyl-3-Buten-1-amine \\
\hline \multirow{11}{*}{ Carnation } & 2-Heptanol \\
\hline & (2-Methyloctyl)benzene \\
\hline & Methyl undecyl ether \\
\hline & N-(2-Furanylmethylene)-3-methyl-1-butanamine \\
\hline & Benzenepropanoic acid, $\beta$-[(tert-butyldimethylsilyl)oxy]-, tert-butyldimethylsilyl ester \\
\hline & 1-Octadecene \\
\hline & 1,2-Nonadecanediol \\
\hline & 1-Undecene \\
\hline & trans-Octahydro-2-(4-methoxyphenyl)-2H-1,3-benzoxazine \\
\hline & 3-chlorophenyl 2-methoxyethyl ester carbonic acid \\
\hline & p-Propylguaiacol \\
\hline \multirow{14}{*}{ Juniper } & $\beta$-Curcumene \\
\hline & Pentamethylcyclopentadiene \\
\hline & $\alpha$-Cadinol \\
\hline & Longifolene-(V4) \\
\hline & trans-calamenene \\
\hline & 1-Ethyl-2,3-dimethyl-piperidin-4-one \\
\hline & Isopinocarveol \\
\hline & Geranyllinalool \\
\hline & Geranyl- $\alpha$-terpinene \\
\hline & Manoyl oxide \\
\hline & 7-Isopropyl-1,1,4a-trimethyl-1,2,3,4,4a,9,10,10a-octahydrophenanthrene \\
\hline & Sclarene \\
\hline & Longifolene-I2 \\
\hline & Hedycaryol \\
\hline \multirow{4}{*}{ Niaouly } & Myrcenol \\
\hline & 1-Undecanol \\
\hline & Undecyl pentafluoropropionate \\
\hline & $\alpha$-Terpinyl acetate \\
\hline \multirow{12}{*}{ Nutmeg } & 3-Isopropenyl-5,5-dimethyl-cyclopentene \\
\hline & 1,4-Dimethyl-4-vinylcyclohexene \\
\hline & Asarone \\
\hline & cis-p-Mentha-2,8-dien-1-ol \\
\hline & cis-p-Menth-2-en-1-ol \\
\hline & 1-Adamantanol \\
\hline & $\beta$-copaene \\
\hline & cis-sesquisabinene hydrate \\
\hline & trans-p-mentha-1(7),8-dien-2-ol \\
\hline & Isomethyleugenol \\
\hline & Guaiol \\
\hline & Isosafrol \\
\hline \multirow{3}{*}{ Oregano } & $\beta$-Terpineol \\
\hline & 2-Octen-1-ol, 3,7-dimethyl-, isobutyrate, (Z)- \\
\hline & N-[4-Bromo-n-butyl]-2-piperidinone \\
\hline
\end{tabular}




\begin{tabular}{|c|c|}
\hline & Bicyclo[3.2.0]hept-2-ene, exo-4-tertbutoxy- \\
\hline & 7,11-Epoxymegastigma-5(6)-en-9-one \\
\hline & $(2 \alpha, 3 \alpha, 5 \beta)-1,1,2$-Trimethyl-3,5-bis(1-methylethenyl)-cyclohexane \\
\hline \multirow{2}{*}{ Rosemary } & p-Menth-2-en-1,4-diol \\
\hline & Humulen-(v1) \\
\hline \multirow{9}{*}{ Mustard } & Diallyl sulfide \\
\hline & Cyclopropylamine \\
\hline & Thiazole \\
\hline & 1-(2,6-Dimethylmorpholin-4-yl)-2-ethyl-hexan-1-one \\
\hline & 3-Butyl-cyclopentanone \\
\hline & 1-Butoxy-2-ethylhexane \\
\hline & Ethyl 14-hydroxy-3,6,9,12-tetraoxatetradecan-1-oate \\
\hline & bis(dimethylhydrazone)ethanedial \\
\hline & 2-Methyl-2-(2,2,4,4-tetramethylpentyl)-aziridine \\
\hline \multirow{4}{*}{ Thyme } & 2-(3,4-Dibromo-4-methylcyclohexyl)propan-2-ol \\
\hline & 1-(1-Methoxymethoxyethyl)cyclohexene \\
\hline & 1-Decylaziridine \\
\hline & Dihydro carveol \\
\hline
\end{tabular}

Finally, Figure 1 shows a histogram of the frequency of most common VOCs. This 38 VOCs represent the ones that are present in at least seven of the oils. The class of compounds most represented is that of terpenes as widely reported in cited literature, with 10 monoterpenes (1R- $\alpha$ pinene, (+)-4-carene, $\beta$-pinene, $\gamma$-terpinene, 4 -carvomenthenol, $\mathrm{M}$-cymene, L- $\alpha$-terpineol, carvacrol, $\beta$-myrcene, camphene) and 1 sesquiterpene (caryophyllene); the other two are alcohols (1-octanal, 2ethylhexanol, 2-methyldecan-1-ol, 1-[1-methyl-2(2-propenyloxy)ethoxy]-2-propanol, 1-dodecanol, 1(isooctyloxy)-2-methyl-2-propanol, 4-methyl-3-heptanol, 1-methoxy-2-propanol) and ether (polypropylene glycol methyl ether, eucalyptol, dipropylene glycol monomethyl ether, ethylene glycol monododecyl ether, diethylene glycol hexyl ether, octyl ether, ethyl glycidyl ether, diethylene glycol monododecyl ether) with 8 compounds each. 


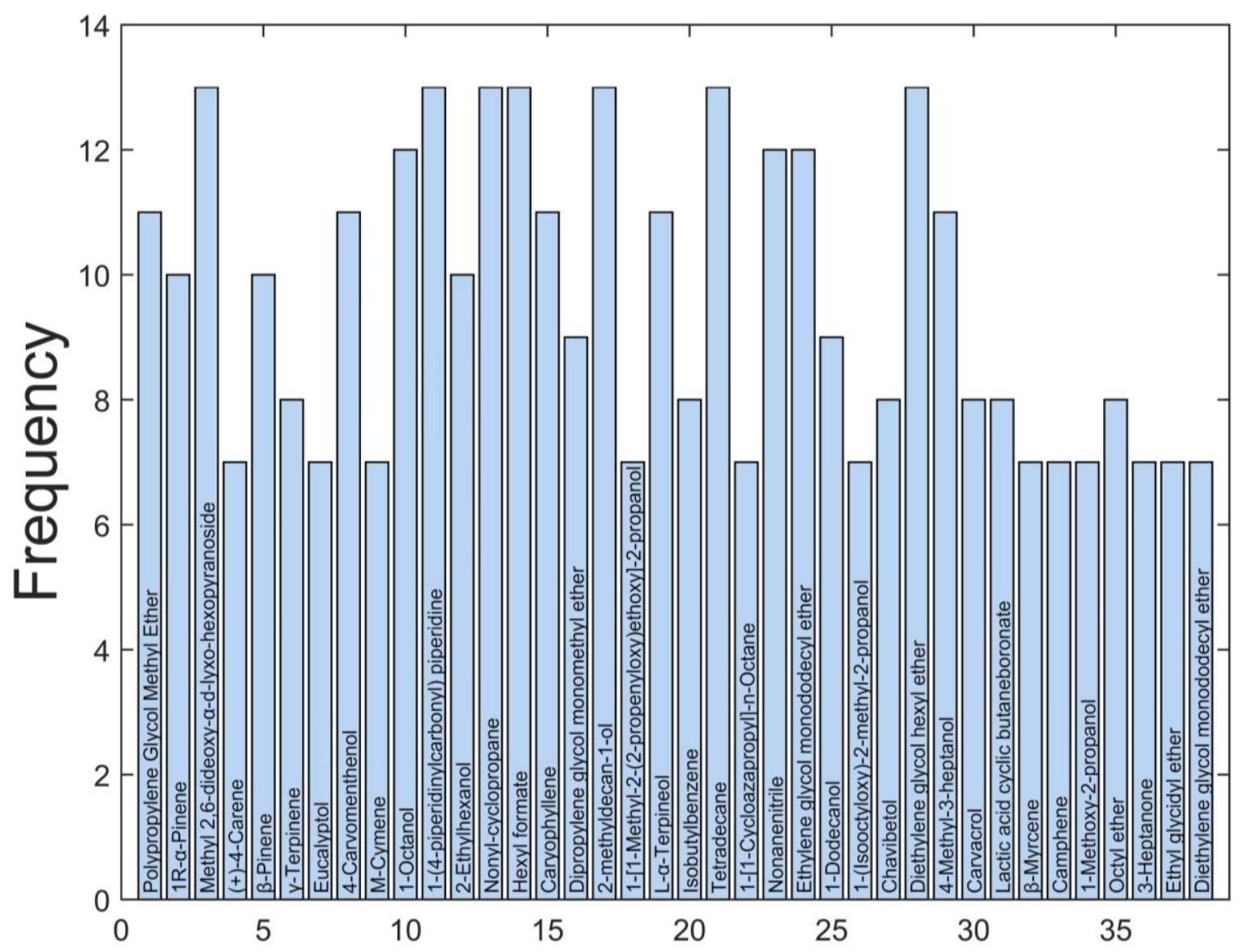

Figure 1. Frequency of the most common compounds among the 13 EOs. 38 are the compounds that appear in at least seven of the oils.

\subsection{S3 Analysis}

Analysis of the data collected with S3 has been carried out using Principal Component Analysis (PCA). In this PCA, features extracted from 7 out of 8 sensors were considered. Indeed, one RGTO SnO2 (heated at $400^{\circ} \mathrm{C}$ ) sensor has been discarded since there was no difference in responses to the different samples. The 2D biplot is shown in Figure 2; it has been done considering the first two principal components (PCs) for a total explained variance equal to 95,45\% (78.27\% for PC1, $17.18 \%$ for PC2). Loadings names are sensors morphology (NW stands for nanowire, RGTO for Rheotaxial Growth and Thermal Oxidation) followed by sensors material. The dotted line separates the two clusters that can be identified: in the upper half-plane juniper, nutmeg, rosemary and mustard samples are situated, the others are in the lower half-plane. The only exception is represented by one measure of coriander that is in the opposite part of the line compared to the others and it is most likely an outlier. 


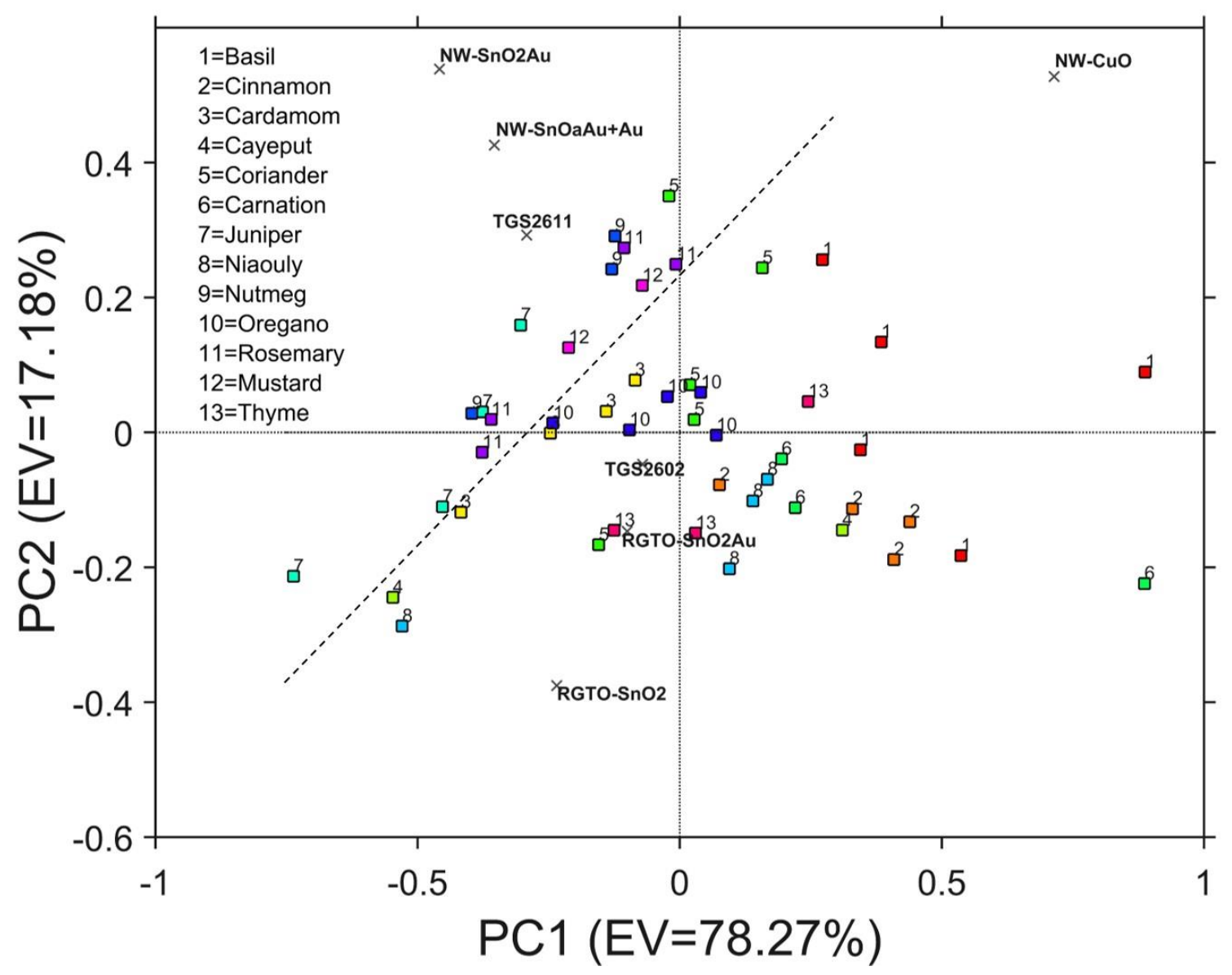

Figure 2. PCA of the first two principal components (total explained variance equal to 95.45\%). Loadings are indicated with crosses, samples with squares and relative number. Legend is in the upper left part of the graph.

To understand which compounds were at the base of the division of the two clusters, Analysis of Variance (ANOVA) has been applied. 7 VOCs had means significantly different between the two group: five of them were characteristic compounds of specific EOs (cyclopropylamine and diallyl sulfide for mustard, 2-Methyloctylbenzene for carnation, 1-Decylaziridine for thyme, N-[2-[pMethoxybenzyl]amino]ethylaziridine for basil), one was common for three EOs (8Methylenepentadecane in cayeout, niaouly and rosemary) and one was found in all the samples (Tetradecane).

Furthermore, an explanation was sought for the fact that there is no clear separation between the different oils. Looking at the common compounds of Figure 1, seven VOCs were found in all the samples. They are methyl 2,6-dideoxy- $\alpha$-d-lyxo-hexopyranoside; 1-(4-piperidinylcarbonyl) piperidine; nonyl-cyclopropane; hexyl formate; 2-methyldecan-1-ol; tetradecane; diethylene glycol hexyl ether. The relative amount of these VOCs compared to the total ranges from $11.7 \%$ to $36.8 \%$. Samples in the upper half-plane of the Figure 2 are characterized by low percentages (from $11.7 \%$ to $14.19 \%)$, except for mustard (36.8\%). However, the fact that mustard is in the same area could be due to its characteristic VOCs. Indeed, they contain different atoms respect to the other EOs, that are nitrogen and sulfur. Samples in the lower half-plane have a higher content of these seven compounds, from $16.2 \%$ to $35.5 \%$. In that case, the EO with the bigger percentage is carnation; however, since an alcohol (2-heptanol) is its most abundant characteristic compound, carnation samples are in the opposite half-plane respect to mustard ones despite the similar amount of compounds in common.

Finally, capability of sensors to distinguish one or more EOs from the others were identified with ANOVA. Hence, the number of times a specific couple of EOs showed significant different means, i.e. recognition by the sensor, has been added. The results are reported in the heatmap below 
(Figure 3), where 0 means that none of the sensors is able to achieve the discrimination and 7 that all of them are capable to do a distinction. All the sensors of the array succeed to identify basil from juniper and six of them basil from cardamom (the only exception is TGS2611). Three nanowire sensors $(\mathrm{CuO}, \mathrm{SnO} 2 \mathrm{Au}+\mathrm{Au}$ and $\mathrm{SnO} 2 \mathrm{Au})$ and both TGS showed the ability to distinguish juniper from cinnamon and carnation. Both tin oxide nanowire and TGS sensors allow the recognition of nutmeg from basil and carnation, of rosemary from basil, cinnamon and carnation, of mustard from basil. Less recognizable EOs are thyme and cayeput (separated only from basil), followed by cardamom and coriander (distinguished from basil, cinnamon and carnation). Conversely, basil, cinnamon and carnation are the most identified oils.

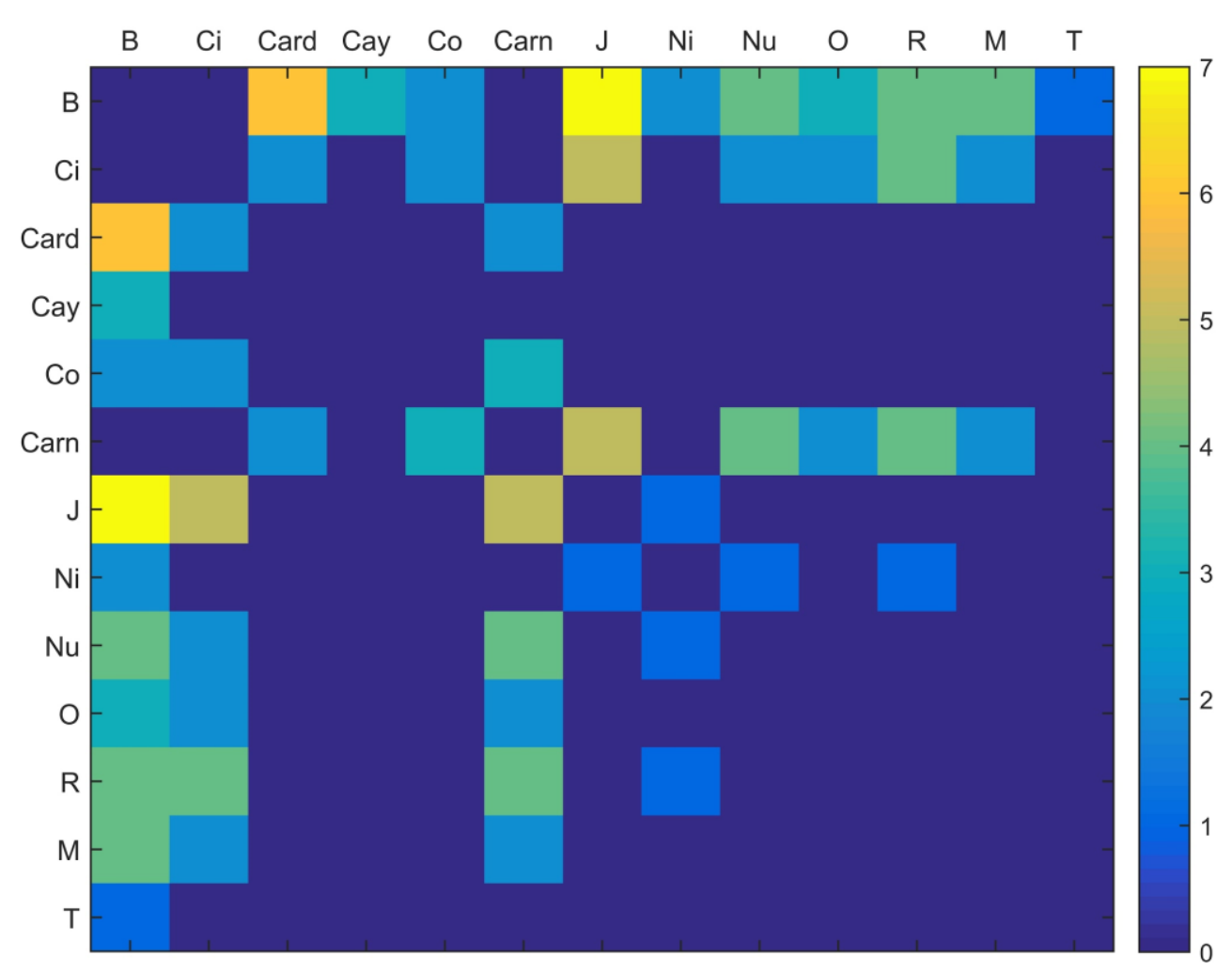

Figure 3. Heatmap regarding the number of sensors useful to discriminate between couples of EOs. B=basil, $\mathrm{Ci}=$ cinnamon, Card=cardamom, Cay=cayeput, $\mathrm{Co}=$ coriander, Carn=carnation, J=Jupiter, Ni=niaouly, $\mathrm{Nu}=$ nutmeg, $\mathrm{O}=$ oregan, $\mathrm{R}=$ rosemary, $\mathrm{M}=$ mustard, $\mathrm{T}=$ thyme.

This preliminary study shows that chemical sensors have potentials to be used to recognize some EOs. In future works, the parameters of analysis could be adjusted to improve the ability of the system to identify more oils. In particular, new methods that allow to highlight the different VOCs and to reduce the influence of common compounds should be put in place.

\section{Materials and Methods}

\subsection{Samples}

Thirteen EOs were considered: basil, cinnamon, cardamom, cayeput, coriander, carnation, juniper, niaouly, nutmeg, oregano, rosemary, mustard, thyme. Of the thirteen EOs, three of the them came from Sigma-Aldrich (Saint Louis, Missouri, USA) and are cardamom, oregano and mustard; the others were bought at Solimè S.r.l. company (Reggio Emilia, Italy). 
Analyzed samples were stored at room temperature, far from sources of heat and light. From each of the thirteen EOs, $37.5 \mu \mathrm{l}$ were taken and put in $20 \mathrm{ml}$ glass vials containing $5 \mathrm{ml}$ of distilled water. The main reason for choosing this concentration was to avoid the saturation of sensor response, due to the high interaction between the amount of VOCs and the sensing material. Hence, the vials were sealed with a metal cap with a PTFE-silicon membrane crimped with an aluminum crimp. For both types of sample, prepared for the measurements with GC-MS and with S3, a method was developed that envisaged optimizing the equilibrium of liquid phase and vapor phase inside the crimped vial, therefore waiting 1 hour before proceeding to the analysis. Two specimens were prepared for GC-MS, while a different number of replicas has been prepared for S3, for a total of 48 measures. The detailed number of samples for each EO is reported in the Table 3.

Table 3. Detailed number of samples for each kind of EOs respectively for each technique used.

\begin{tabular}{|c|c|c|c|}
\hline $\mathbf{N}^{\circ}$ & EOs Name & $\begin{array}{c}\text { GC-MS } \\
\mathbf{N}^{\circ} \text { of Samples }\end{array}$ & $\begin{array}{c}\mathrm{S} 3 \\
\mathrm{~N}^{\circ} \text { of Samples }\end{array}$ \\
\hline 1 & Basil & 2 & 5 \\
\hline 2 & Cinnamon & 2 & 4 \\
\hline 3 & Cardamom & 2 & 4 \\
\hline 4 & Cayeput & 2 & 2 \\
\hline 5 & Coriander & 2 & 5 \\
\hline 6 & Carnation & 2 & 3 \\
\hline 7 & Juniper & 2 & 4 \\
\hline 8 & Niaouly & 2 & 4 \\
\hline 9 & Nutmeg & 2 & 3 \\
\hline 10 & Oregano & 2 & 5 \\
\hline 11 & Rosemary & 2 & 4 \\
\hline 12 & Mustard & 2 & 2 \\
\hline 13 & Thyme & 2 & 3 \\
\hline Total & & 26 & 48 \\
\hline
\end{tabular}

\subsection{GC-MS Analysis}

The Gas Chromatograph (GC) used during the analyses was a Shimadzu GC2010 PLUS (Kyoto, KYT, Japan), equipped with a Shimadzu single quadrupole Mass Spectometer (MS) MSQP2010 Ultra (Kyoto, KYT, Japan) and an autosampler HT280T (HTA s.r.l., Brescia, Italy). The GCMS analysis was coupled with the Solid-Phase Micro Extraction (SPME) method in order to find the most characteristic VOCs for each sample.

The fiber used for the adsorption of volatiles was a DVB/CAR/PDMS-50/30 $\mu \mathrm{m}$ (Supelco Co. Bellefonte, PA, USA). The fiber was exposed to the headspace of the vials after heating and shaking 
the samples in the HT280T oven, thermostatically regulated at $50{ }^{\circ} \mathrm{C}$ for $15 \mathrm{~min}$, with the aim of creating the headspace equilibrium. The length of the fiber in the headspace was kept constant. Desorption of volatiles took place in the injector of the GC-MS for 6 min at $250{ }^{\circ} \mathrm{C}$.

The gas chromatograph was operated in the direct mode throughout the run, with the mass spectrometer in electron ionization (EI) mode $(70 \mathrm{eV})$. GC separation was performed on a MEGAWAX capillary column $(30 \mathrm{~m} \times 0.25 \mathrm{~mm} \times 0.25 \mu \mathrm{m}$, Agilent Technologies, Santa Clara, CA, USA). Ultrapure helium $(99.99 \%)$ was used as the carrier gas, at the constant flow rate of $1.3 \mathrm{~mL} / \mathrm{min}$. The following GC oven temperature programming was applied. At the beginning, the column was held at $40{ }^{\circ} \mathrm{C}$ for $8 \mathrm{~min}$, and then raised from 40 to $190{ }^{\circ} \mathrm{C}$ at $4{ }^{\circ} \mathrm{C} / \mathrm{min}$; then, the temperature was maintained at $190{ }^{\circ} \mathrm{C}$ for $5 \mathrm{~min}$. Next, the temperature was raised from $190{ }^{\circ} \mathrm{C}$ to $210{ }^{\circ} \mathrm{C}$, with a rate of $5{ }^{\circ} \mathrm{C} / \mathrm{min}$; finally, $210^{\circ} \mathrm{C}$ was maintained for $5 \mathrm{~min}$.

The GC-MS interface was kept at $200^{\circ} \mathrm{C}$. The mass spectra were collected over the range of 45 to $500 \mathrm{~m} / \mathrm{z}$ in the Total Ion Current (TIC) mode, with scan intervals at $0.3 \mathrm{~s}$. The identification of the volatile compounds was carried out using the NIST11 and the FFNSC2 libraries of mass spectra.

\subsection{S3 Analysis}

S3 device used in the present work has been completely designed and constructed at SENSOR Laboratory (University of Brescia, Italy) in collaboration with NASYS S.r.l., a spin-off of the University of Brescia. It has been described in other works [39,40-45,46]. Briefly, the tool comprises three parts: pneumatic components, that transfer VOCs from the head-space of samples to the sensing chamber; electronic boards, that manage the acquisition and transmission of the data from the device to the dedicated Web-App and allow the synchronization between S3 and the auto-sampler; sensing chamber, that can host from five to ten different MOX gas sensors and is thermostated and isolated in order to avoid any influence of the surrounding environment. To function properly, the sensors need a reference value, which has been obtained by filtering the ambient air with a small metal cylinder $(21.5 \mathrm{~cm}$ in length, $5 \mathrm{~cm}$ in diameter) filled with activated carbons.

Eight MOX gas sensors were used. Three of them are MOX nanowire, as presented in References [34]. Two of them are tin oxides nanowires sensors, both grown by means of the Vapor Liquid Solid technique [47], using a gold catalyst on the alumina substrate and functionalizing one of them with gold clusters; the third sensor has an active layer of copper oxide nanowires. The working temperature is $350{ }^{\circ} \mathrm{C}, 350{ }^{\circ} \mathrm{C}$ and $400{ }^{\circ} \mathrm{C}$, respectively. The other three sensors are prepared with RGTO thin film technology [48]; one is tin oxide functionalized with gold clusters (working at 400 ${ }^{\circ} \mathrm{C}$ ), while the other two are pure tin oxide (working at $300{ }^{\circ} \mathrm{C}$ and at $400{ }^{\circ} \mathrm{C}$, respectively). The last two are commercial MOX sensors produced by Figaro Engineering Inc. (Osaka, Japan). In particular, they are the TGS2611 and TGS2602, which are sensitive to natural gases and odorous gases like ammonia, respectively, according to the datasheet of the company. Commercial sensors have been mounted on our e-nose in order to evaluate the performances of nanowire sensors. Details of S3 sensors made at SENSOR Laboratory are summarized in Table 4. Response to $5 \mathrm{ppm}$ of ethanol, selectivity (response ethanol/response carbon monoxide) and limit of detection (LOD) of ethanol are also included.

Table 4. Type, composition, morphology, operating temperature, response $(\Delta R / R)$, selectivity (response ethanol/response carbon monoxide) and limit of detection (LOD) of ethanol for S3 sensors made at the SENSOR Laboratory.

\begin{tabular}{|c|c|c|c|c|c|c|}
\hline $\begin{array}{l}\text { Materials } \\
\text { (Type) }\end{array}$ & Composition & $\begin{array}{c}\text { Morpholog } \\
\mathbf{y}\end{array}$ & $\begin{array}{c}\text { Operating } \\
\text { Temperature }\left({ }^{\circ} \mathrm{C}\right)\end{array}$ & $\begin{array}{l}\text { Response to } 5 \\
\text { ppm of Ethanol }\end{array}$ & $\begin{array}{c}\text { Selectivit } \\
\mathbf{y}\end{array}$ & $\begin{array}{c}\text { Limit of Detection } \\
\text { (LOD) of Ethanol (ppm) }\end{array}$ \\
\hline $\mathrm{SnO}_{2} \mathrm{Au}(\mathrm{n})$ & $\begin{array}{c}\mathrm{SnO}_{2} \text { functionalized } \\
\text { with Au clusters }\end{array}$ & RGTO & $400^{\circ} \mathrm{C}$ & 6.5 & 3 & 0.5 \\
\hline $\mathrm{SnO}_{2}(\mathrm{n})$ & $\mathrm{SnO}_{2}$ & RGTO & $300^{\circ} \mathrm{C}$ & 3.5 & 2.5 & 1 \\
\hline $\mathrm{SnO}_{2}(\mathrm{n})$ & $\mathrm{SnO}_{2}$ & RGTO & $400^{\circ} \mathrm{C}$ & 4 & 2 & 0.8 \\
\hline $\mathrm{SnO}_{2} \mathrm{Au}+\mathrm{Au}(\mathrm{n})$ & $\begin{array}{c}\mathrm{SnO}_{2} \text { grown with } \mathrm{Au} \\
\text { and functionalized } \\
\text { with gold clusters }\end{array}$ & Nanowire & $350^{\circ} \mathrm{C}$ & 7 & 2.5 & 0.5 \\
\hline $\mathrm{SnO}_{2} \mathrm{Au}(\mathrm{n})$ & $\mathrm{SnO}_{2}$ grown with $\mathrm{Au}$ & Nanowire & $350^{\circ} \mathrm{C}$ & 5 & 2.1 & 1 \\
\hline $\mathrm{CuO}(\mathrm{p})$ & $\mathrm{CuO}$ & Nanowire & $400^{\circ} \mathrm{C}$ & 1.5 & 1.5 & 1 \\
\hline
\end{tabular}


The auto-sampler head-space system HT2010H was coupled with S3. It supports a 42-loadingsites carousel and a shaking oven to equilibrate the sample head-space. The vials were placed in a randomized mode into the carousel. Each vial was incubated at $40{ }^{\circ} \mathrm{C}$ for $5 \mathrm{~min}$ in the auto-sampler oven and shaken every $6 \mathrm{~s}$ for $12 \mathrm{~s}$ during the incubation. The sample head-space was then extracted from the vial in the dynamic head-space path and released into the carried flow $(50 \mathrm{sccm})$. The analysis timeline can be divided into three different steps for a duration of $600 \mathrm{~s}$ (10 $\mathrm{min}$ ) per sample, $60 \mathrm{~s}$ to analyze samples and $510 \mathrm{~s}$ to restore the base line. Thanks to the processor integrated in the S3 instrument, the frequency at which the equipment works is equal to $1 \mathrm{~Hz}$.

\subsection{Data Analysis}

Statistical techniques have been applied to extract information from S3 data. PCA has been performed to understand if the array of sensors was able to discriminate among the EOs. Hence, $\Delta R / R$ feature has been calculated for the eight sensors as input variables. Sequentially, ANOVA has been used to interpret grouping of the EOs on the 2D biplot and to individuate best sensors for EOs discrimination, selecting a significance level equal to 0.05 . Finally, multiple comparison of the ANOVA results has been done using Tukey's honest significance test.

\section{Conclusions}

Thanks to this detailed study, it has been possible to reach and obtain different important points for the future development of this type of research. Especially as regards the results obtained with the GC-MS, it has been highlighted that 95 out of 204 VOCs characterize one oil from the others. As far as $\mathrm{S} 3$ is concerned, the results obtained and the conclusions that we can draw from it can be further divided into two parts, a part concerning the whole array and a part related to the responses of the individual sensors to the analyzed oils. Through PCA, it can be suggested that S3 was able to distinguish between two cluster: one is formed by juniper, nutmeg, rosemary and mustard, the other by basil, cinnamon, cardamom, cayeput, coriander, carnation, niaouly, oregano and thyme. From the analysis of individual sensors, it turned out that basil, cinnamon and carnation are the most identifiable oils with different number and typology of sensors, especially tin oxide and copper oxide nanowires, while cayeput and thyme are more mistakable samples.

Author Contributions: These authors contributed equally to this work.

Funding: This research received no external funding.

Conflicts of Interest: The authors declare no conflict of interest.

\section{References}

1. Asbahani, A. El; Miladi, K.; Badri, W.; Sala, M.; Addi, E. H. A.; Casabianca, H.; Mousadik, A. El; Hartmann, D.; Jilale, A.; Renaud, F. N. R.; Elaissari, A.; Lyon, C. B. Essential oils : From extraction to encapsulation. Int. J. Pharm. 2015, 483, 220-243, doi:10.1016/j.ijpharm.2014.12.069.

2. Okoh, O. O.; Sadimenko, A. P.; Afolayan, A. J. Comparative evaluation of the antibacterial activities of the essential oils of Rosmarinus officinalis L . obtained by hydrodistillation and solvent free microwave extraction methods. Food Chem. 2010, 120, 308-312, doi:10.1016/j.foodchem.2009.09.084.

3. Ferhat, M. A.; Meklati, B. Y.; Chemat, F. Comparison of different isolation methods of essential oil from Citrus fruits: cold pressing, hydrodistillation and microwave " dry " distillation. 2007, 494-504, doi:10.1002/ffj.

4. Etenate, A. D. J. P.; Eireles, M. A. N. A. M. Supercritical Extraction of Essential Oil from Aniseed ( Pimpinella anisum L ) Using CO 2 : Solubility , Kinetics, and Composition Data. 2003, 1518-1523.

5. Braga, M. E. M.; Ehlert, P. A. D.; Ming, L. C.; Meireles, M. A. A. Supercritical fluid extraction from Lippia alba: global yields, kinetic data, and extract chemical composition. 2005, 34, 149-156, doi:10.1016/j.supflu.2004.11.008.

6. Dima, C.; Dima, S. ScienceDirect Essential oils in foods : extraction, stabilization, and toxicity. Curr. Opin. Food Sci. 5, 29-35, doi:10.1016/j.cofs.2015.07.003. 
7. Bakkali, F.; Idaomar, M. Biological effects of essential oils - A review. 2008, 46, 446-475, doi:10.1016/j.fct.2007.09.106.

8. Mahato, N.; Sharma, K.; Koteswararao, R.; Sinha, M.; Raj, E. Citrus essential oils: Extraction , authentication and application in food preservation. 2017, 8398, doi:10.1080/10408398.2017.1384716.

9. Roman, K.; Flasin, M. Essential oils as food eco-preservatives : Model system studies on the effect of temperature on limonene antibacterial activity. 2017, 235, 127-135, doi:10.1016/j.foodchem.2017.05.051.

10. Noshirvani, N.; Ghanbarzadeh, B.; Gardrat, C. Food Hydrocolloids Cinnamon and ginger essential oils to improve antifungal, physical and mechanical properties of chitosan-carboxymethyl cellulose fi lms. 2017, 70, 36-45, doi:10.1016/j.foodhyd.2017.03.015.

11. Melo, D.; Ribeiro-santos, R.; Andrade, M. Trends in Food Science \& Technology Use of essential oils in active food packaging: Recent advances and future trends. 2017, 61, 132-140, doi:10.1016/j.tifs.2016.11.021.

12. Severino, R.; Ferrari, G.; Dang, K.; Donsì, F. Antimicrobial effects of modi fi ed chitosan based coating containing nanoemulsion of essential oils, modi fi ed atmosphere packaging and gamma irradiation against Escherichia coli O157 : H7 and Salmonella Typhimurium on green beans. 2015, 50, 215-222, doi:10.1016/j.foodcont.2014.08.029.

13. Tongnuanchan, P.; Benjakul, S. Essential Oils: Extraction, Bioactivities, and Their Uses for Food Preservation. 2014, 79, 1231-1249, doi:10.1111/1750-3841.12492.

14. Omonijo, F. A.; Ni, L.; Gong, J.; Wang, Q.; Lahaye, L.; Yang, C. Essential oils as alternatives to antibiotics in swine production. Anim. Nutr. 2018, 4, 126-136, doi:10.1016/j.aninu.2017.09.001.

15. Zhai, H.; Liu, H.; Wang, S.; Wu, J.; Kluenter, A. Potential of essential oils for poultry and pigs. Anim. Nutr. 2018, 4, 179-186, doi:10.1016/j.aninu.2018.01.005.

16. Oliveira, J. De; Alves, K.; Souza, D.; Carolina, A.; Vital, P.; Cunha, V.; Favoreto, K.; Nunes, I. Clove and rosemary essential oils and encapsuled active principles (eugenol, thymol and vanillin blend) on meat quality of feedlot-finished heifers. Meat Sci. 2017, 130, 50-57, doi:10.1016/j.meatsci.2017.04.002.

17. Rivera, J.; Crandall, P. G.; Bryan, C. A. O.; Ricke, S. C. Essential oils as antimicrobials in food systems e A review. Food Control 2015, 54, 111-119, doi:10.1016/j.foodcont.2014.12.040.

18. Compositions, C.; Activities, A. Cardamom, Cumin, and Dill Weed Essential Oils: Chemical Compositions, Antimicrobial Activities, and Mechanisms of Action against Campylobacter spp. 2017, doi:10.3390/molecules22071191.

19. Sa, O. Sensitivity of four pathogenic bacteria to Turkish thyme and oregano hydrosols. 2003, 36, 467473, doi:10.1016/S0023-6438(03)00037-9.

20. Sakkas, H.; Papadopoulou, C. Antimicrobial Activity of Basil, Oregano, and Thyme Essential Oils. 2016, doi:10.4014/jmb.1608.08024.

21. Singh, S.; Singh, G.; Catalan, C. A. Comparative Studies of Chemical Composition, Antioxidant and Antimicrobial Potentials of Essential Oils and Oleoresins Obtained from Seeds Toxicology : Open Access Comparative Studies of Chemical Composition, Antioxidant and Antimicrobial Potentials of Essential Oils and Oleoresins Obtained from Seeds and Leaves of. 2017, doi:10.4172/2476-2067.1000119.

22. Khalil, N.; Ashour, M.; Fikry, S.; Naser, A.; Salama, O. Future Journal of Pharmaceutical Sciences Chemical composition and antimicrobial activity of the essential oils of selected Apiaceous fruits. Futur. J. Pharm. Sci. 2018, 4, 88-92, doi:10.1016/j.fjps.2017.10.004.

23. Zheljazkov, V. D.; Kacaniova, M.; Dincheva, I.; Radoukova, T.; Semerdjieva, I. B.; Astatkie, T.; Schlegel, V. Industrial Crops \& Products Essential oil composition, antioxidant and antimicrobial activity of the galbuli of six juniper species. Ind. Crop. Prod. 2018, 124, 449-458, doi:10.1016/j.indcrop.2018.08.013.

24. Pandey, S.; Tiwari, S.; Kumar, A.; Niranjan, A.; Chand, J.; Lehri, A. Industrial Crops \& Products Antioxidant and anti-aging potential of Juniper berry ( Juniperus communis L .) essential oil in Caenorhabditis elegans model system. Ind. Crop. Prod. 2018, 120, 113-122, doi:10.1016/j.indcrop.2018.04.066.

25. Raut, J. S.; Karuppayil, S. M. A status review on the medicinal properties of essential oils. Ind. Crop. Prod. 2014, 62, 250-264, doi:10.1016/j.indcrop.2014.05.055.

26. Veum, K., Sudduth, K., Kremer, R., Kitchen, N. Sensor data fusion for soil health assessment. Geoderma 2017, 305, 53-61. doi:10.1016/j.geoderma.2017.05.031.

27. Ullah, N., Mansha, M., Khan, I., Qurashi, A. Nanomaterial-based optical chemical sensors for the detection of heavy metals in water: Recent advances and challenges. Trends in Analytical Chemistry 2018, 100, 155-166. doi:10.1016/j.trac.2018.01.002.

28. Das, S., Saha, P. A review of some advanced sensors used for health diagnosis of civil engineering structures. Measurement 2018, 129, 68-90. doi:10.1016/j.measurement.2018.07.008.

29. Gebicki, J., Dymerski, T. Application of Chemical Sensors and Sensor Matrixes to Air Quality Evaluation. 
Comprehensive Analytical Chemistry 2016, 73, 267-294. doi:10.1016/bs.coac.2016.02.007.

30. Kassal, P., Steinberg, M., Steinberg, I.M. Wireless chemical sensors and biosensors: A review. Sensors and Actuators B: Chemical 2018, 266, 228-245. doi:10.1016/j.snb.2018.03.074.

31. Yang, Q., Li, J., Wang, X., Peng, H., Xiong, H., Chen, L. Strategies of molecular imprinting-based fluorescence sensors for chemical and biological analysis. Biosensors and Bioelectronics 2018, 112, 54-71. doi:10.1016/j.bios.2018.04.028.

32. Correa, D., Pavinatto, A., Mercante, L.A., Mattoso, L.H.C., Oliveira, J.E., Riul, A. Chemical sensors based on hybrid nanomaterials for food analysis. Nanobiosensors 2017, 205-244. doi:10.1016/B978-0-12-8043011.00006-0.

33. Pavase, T.R., Lin, H., Shaikh, Q., Hussain, S., Li, Z., Ahmed, I., Lv, L., Sun, L., Shah, S.B.H., Kalhoro, M.T. Recent adbances of conjugated polymer (CP) nanocomposite-based chemical sensors and their applications in food spoilage detection: A comprehensive review. Sensors and Actuators B: Chemical 2018, 273, 1113-1138. doi:10.1016/j.snb.2018.06.118.

34. Sberveglieri, G.; Concina, I.; Comini, E.; Falasconi, M.; Ferroni, M.; Sberveglieri, V. Synthesis and integration of tin oxide nanowires into an electronic nose. Vacuum 2012, 86, 532-535, doi:10.1016/j.vacuum.2011.10.004.

35. Ponzoni, A., Baratto, C., Cattabiani, N., Falasconi, M., Galstyan, V., Nunez-Carmona, E., Rigoni, F., Sberveglieri, V., Zambotti, G., Zappa, D. Metal Oxide Gas Sensors, a Survey of Selectivity Issues Addressed at the SENSOR Lab, Brescia (Italy). Sensors 2017, 4. doi:10.3390/s17040714.

36. Núñez Carmona, E., Sberveglieri, V., Comini, E., Zappa, D., Pulvirenti, A. Nanowire Technology for the Detection of Microorganisms in Potable Water. Procedia Engineering 2014, 87, 1453-1456. doi:10.1016/j.proeng.2014.11.723.

37. Sberveglieri, V., Núñez Carmona, E., Pulvirenti, A. Detection of Microorganism in Water and Different Food Matrix by Electronic Nose. In: Mason A., Mukhopadhyay S., Jayasundera K. (eds) Sensing Technology: Current Status and Future Trends III. Smart Sensors, Measurement and Instrumentation, vol 11. Springer, Cham. doi:10.1007/978-3-319-10948-0_12.

38. Zambotti, G., Sberveglieri, V., Gobbi, E., Falasconi, M., Nunez, E., Pulvirenti, A. Fast Identification of MIcrobiological COntamination in Vegetable Soup by Electroni Nose. Procedia Engineering 2014, 87, 13021305. doi:10.1016/j.proeng.2014.11.686.

39. Abbatangelo, M.; Sberveglieri, V. Application of a Novel S3 Nanowire Gas Sensor Device in Parallel with GC-MS for the Identification of Rind Percentage of Grated Parmigiano Reggiano., doi:10.3390/s18051617.

40. Abbatangelo, M.; Núñez-carmona, E.; Sberveglieri, V. Application of a novel S3 nanowire gas sensor device in parallel with GC-MS for the identi fi cation of Parmigiano Reggiano from US and European competitors. J. Food Eng. 2018, 236, 36-43, doi:10.1016/j.jfoodeng.2018.05.009.

41. Adam, M.; Juklov, M.; Bajer, T.; Eisner, A.; Ventura, K. Comparison of three different solid-phase microextraction fibres for analysis of essential oils in yacon ( Smallanthus sonchifolius ) leaves. 2005, 1084, 2-6, doi:10.1016/j.chroma.2005.05.072.

42. Cagliero, C.; Bicchi, C.; Cordero, C.; Liberto, E.; Rubiolo, P.; Sgorbini, B. Analysis of essential oils and fragrances with a new generation of highly inert gas chromatographic columns coated with ionic liquids. J. Chromatogr. A 2017, 1495, 64-75, doi:10.1016/j.chroma.2017.03.029.28.

43. Adams, P.R. Identification of Essential Oil Components by Gas Cromatography/Mass Spectrometry (ed 4.1), 2017.

44. Zenkevich, I.G. Retention Indices for Frequently Reported Compounds of Plant Essential Oils. Journal of Physical and Chemical Reference Data 2011, 40. doi:10.1063/1.3653552.

45. Sberveglieri, V. Validation of Parmigiano Reggiano Cheese Aroma Authenticity, Categorized through the Use of an Array of Semiconductors Nanowire Device (S3). Materials 2016, 82. doi:10.3390/ma9020081.

46. Sberveglieri, V., Bhandari, M.P., Núñez Carmona, E., Betto, G., Sberveglieri, G. A Novel MOS Nanowire Gas Sensor Device (S3) and GC-MS-Based Approach for the Characterization of Grated Parmigiano Reggiano Cheese. Biosensors 2016, 60. doi:10.3390/bios6040060.

47. Wagner, R. S.; Ellis, W. C. Vapor-liquid-solid mechanism of single crystal growth. 2011, 89, 4-6, doi:10.1063/1.1753975.

48. Sberveglieri, G. Recent developments in semiconducting thin-film gas sensors. Sensors and Actuators B: Chemical 1995, 23, 103-109. doi:10.1016/0925-4005(94)01278-P. 